OPEN ACCESS

Edited by:

Uday Kishore,

Brunel University London,

United Kingdom

Reviewed by:

Kushagra Bansal,

Harvard Medical School,

United States

Juni Xing,

Houston Methodist Research

Institute, United States

Taruna Madan,

National Institute for Research

in Reproductive Health

(ICMR), India

${ }^{*}$ Correspondence:

Till Adhikary

adhikary@imt.uni-marburg.de

Specialty section:

This article was submitted to Molecular Innate Immunity,

a section of the journal

Frontiers in Immunology

Received: 23 December 2017 Accepted: 08 June 2018

Published: 27 June 2018

Citation:

Unger A, Finkernagel F, Hoffmann N, Neuhaus F, Joos B, Nist A, Stiewe T, Visekruna A, Wagner U, Reinartz S, Müller-Brüsselbach S, Müller $R$ and Adhikary T (2018) Chromatin Binding of c-REL and p65 Is Not Limiting for

Macrophage IL12B Transcription

During Immediate Suppression by

Ovarian Carcinoma Ascites.

Front. Immunol. 9:1425.

doi: 10.3389/fimmu.2018.01425

\section{Chromatin Binding of $c-R E L$ and p65 Is Not Limiting for Macrophage IL12B Transcription During Immediate Suppression by Ovarian Carcinoma Ascites}

Annika Unger ${ }^{1}$, Florian Finkernagel ${ }^{1}$, Nathalie Hoffmann ${ }^{2}$, Felix Neuhaus ${ }^{1}$, Barbara Joos ${ }^{1}$, Andrea Nist ${ }^{3}$, Thorsten Stiewe ${ }^{3}$, Alexander Visekruna ${ }^{4}$, Uwe Wagner ${ }^{5}$, Silke Reinartz ${ }^{6}$, Sabine Müller-Brüsselbach ${ }^{1}$, Rolf Müller ${ }^{1}$ and Till Adhikary ${ }^{1 *}$

'Institute for Molecular Biology and Tumor Research (IMT), Center for Tumor Biology and Immunobiology (ZTI), Philipps University of Marburg, Marburg, Germany, ${ }^{2}$ Experimental Tumor Research Group, Center for Tumor Biology and Immunobiology (ZTI), Philipps University of Marburg, Marburg, Germany, ${ }^{3}$ Genomics Core Facility, ZTI, Philipps University of Marburg, Marburg, Germany, ${ }^{4}$ Institute for Medical Microbiology and Hygiene, Biomedical Research Center (BMFZ), Philipps University of Marburg, Marburg, Germany, ${ }^{5}$ Clinic for Gynecology, Gynecological Oncology and Gynecological Endocrinology, Philipps University of Marburg, Marburg, Germany, ${ }^{6}$ Clinic for Gynecology, Gynecological Oncology and Gynecological Endocrinology, ZTI, Philipps University of Marburg, Marburg, Germany

Tumors frequently exploit homeostatic mechanisms that suppress expression of IL-12, a central mediator of inflammatory and anti-tumor responses. The p40 subunit of the IL-12 heterodimer, encoded by IL $12 B$, is limiting for these functions. Ovarian carcinoma patients frequently produce ascites which exerts immunosuppression by means of soluble factors. The NFKB pathway is necessary for transcription of $I L 12 B$, which is not expressed in macrophages freshly isolated from ascites. This raises the possibility that ascites prevents $I L 12 B$ expression by perturbing NFKB binding to chromatin. Here, we show that ascites-mediated suppression of IL12B induction by LPS plus IFN $\gamma$ in primary human macrophages is rapid, and that suppression can be reversible after ascites withdrawal. Nuclear translocation of the NFKB transcription factors $c$-REL and p65 was strongly reduced by ascites. Surprisingly, however, their binding to the IL12B locus and to CXCL10, a second NFKB target gene, was unaltered, and the induction of CXCL10 transcription was not suppressed by ascites. These findings indicate that, despite its reduced nuclear translocation, NFkB function is not generally impaired by ascites, suggesting that ascites-borne signals target additional pathways to suppress IL12B induction. Consistent with these data, IL-10, a clinically relevant constituent of ascites and negative regulator of $\mathrm{NF \kappa B}$ translocation, only partially recapitulated $I L 12 B$ suppression by ascites. Finally, restoration of a defective IL-12 response by appropriate culture conditions was observed only in macrophages from a subset of donors, which may have important implications for the understanding of patient-specific immune responses.

Keywords: IL12B, NFKB, REL, p65, ascites, ovarian carcinoma, macrophages, immunosuppression 


\section{INTRODUCTION}

Solid tumors are frequently accompanied by large numbers of tumor-associated macrophages (TAMs), and their abundance is correlated with poor prognosis in several tumor types $(1,2)$. Advanced ovarian carcinoma (OC) often coincides with considerable accumulation of a malignant peritoneal effusion termed ascites which harbors large numbers of floating stromal and tumor cells. Its immunosuppressive properties are at least in part conferred by soluble mediators $(3,4)$. Like their counterparts from other tumor entities, TAMs isolated from OC ascites display an anti-inflammatory phenotype including high expression of the hemoglobin scavenger receptor CD163 (5). In follow-up studies, we found that the transcriptomes of ovarian carcinoma TAMs and those of peritoneal macrophages from non-tumor patients are very similar with the exception of a set of genes involved in extracellular matrix reorganization (6), which is a hallmark of wound healing and tumorigenesis. The expression of this gene set is correlated with poor survival $(6,7)$. On the other hand, elevated expression of an interferon (IFN)-inducible gene set in TAMs is correlated with improved survival (7). A recent metaanalysis of TAM studies in OC patients (8) reflects the findings that $\mathrm{CD} 163$ expression correlates with worse prognosis, while pro-inflammatory macrophage polarization is positively associated with survival.

The ability of human macrophages to produce nitric oxide has been debated extensively (9-14). Apparently, their cytotoxic activity is rather exerted indirectly via secretion of cytokines, especially interleukin-12 (IL-12), which activates cytotoxic functions of T and NK cells (15-19). IL-10 is an immunosuppressive cytokine present in large amounts in OC ascites (20), and there is a strong negative correlation of its level with patient survival $(5,21)$. In the ovarian tumor microenvironment, IL10 is expressed predominantly by TAMs $(3,21,22)$. A critical function of IL-10 is to repress transcription of $\operatorname{IL12B}(23,24)$, which encodes for the p40 subunit of IL-12 and IL-23 that is limiting for heterodimer formation. Antigen-presenting cells are the main producers of IL-12p40 (25). On the other hand, IL-12 represses IL10 transcription. This reciprocal blockade, "the IL-10-IL-12 circuit" (26), is enforced by positive feedback of IL-10 and IL-12 production, respectively (27). Mechanisms involved in these positive feedback loops are interdependent upregulations of IL-10 and CD163 (28-30) or, vice versa, instigation of IFN $\gamma$ production by $\mathrm{T}$ and NK cells upon exposure to IL-12 (31-33); in turn, IFN $\gamma$

\footnotetext{
Abbreviations: APC, antigen-presenting cell; BMDM, bone marrow-derived macrophage; $\mathrm{CD}$, cluster of differentiation; C/EBP, CCAAT/enhancer binding protein; ChIP, chromatin immunoprecipitation; ENCODE, encyclopedia of DNA elements; GEO, gene expression omnibus; IFN, interferon; IKK, IкB kinase; IL, interleukin; $\mathrm{I} \kappa \mathrm{B}$, inhibitor of $\kappa$ light chain enhancer of activated B cells; JAK, Janus kinase; LPS, lipopolysaccharide; MDM, monocyte-derived macrophage; MFI, mean fluorescence intensity; MIRA, methylated CpG island recovery assay; MSMD, Mendelian susceptibility to mycobacterial disease; NFкB, nuclear factor $\kappa$ light chain enhancer of activated B cells; NK, natural killer; OC, ovarian carcinoma; PBMC, peripheral blood mononuclear cell; pMDM, monocyte-derived macrophage from a patient; REL, reticuloendotheliosis; RPL27, ribosomal protein 27, large subunit; RPMI, Roswell Park Memorial Institute; RRID, research resource identifier; STAT, signal transducer and activator of transcription; TAM, tumor-associated macrophage; Th, T helper; TSS, transcription start site.
}

enables production of IL-12 by monocytic cells. Indeed, it was shown that IFNy is capable of relieving suppression of IL-12 production by OC ascites $(7,34)$ and is, therefore, required for the pro-inflammatory feedback loop. Taken together, switching between IL-10 and IL-12 production can toggle between the anti-inflammatory, immunosuppressive Th2 state, and proinflammatory, tumoricidal Th1 activation (23). The large number of immunosuppressive TAMs relative to other hematopoietic cells in $\mathrm{OC}$ ascites provides them with a decisive, pro-tumorigenic role in the microenvironment which at least in part depends on efficient suppression of $I L 12 B$ transcription (4).

IL12B transcription is induced cooperatively by STAT1 and the NFKB pathway (27). Stimuli that activate each of these have antitumorigenic effects in vivo $(7,35)$. A critical role for the IFN $\gamma$ pathway in $I L 12 B$ expression is highlighted by mutations that cause autosomal Mendelian susceptibility to mycobacterial disease (MSMD). These were reported to occur in seven genes: IL12B, IL12RB1, IFNGR1, IFNGR2, IRF8, ISG15, and STAT1 (36-38). IFN $\gamma$, if present during macrophage differentiation in OC ascites in vitro, can override suppression of IL-12 production $(7,34)$. Therefore, gene regulation by IFN $\gamma$ and STAT1 is generally functional in macrophages exposed to ascites. Moreover, pretreatment of macrophages with IFN $\gamma$ can prime them for stimulus-dependent IL-12 production (39), indicating that an IFN $\gamma$-mediated effect can limit the amplitude of the response.

The NFKB transcription factor $c$-REL (from now on abbreviated as REL), which is predominantly expressed in hematopoietic cells $(40,41)$, is crucial for $I L 12 B$ expression $(42,43)$. The requirement for $\mathrm{NF \kappa B}$ is underscored by the finding that mutations in NEMO/IKKG which disrupt induction of IL12B via CD40-IKK $\gamma$ cause X-linked MSMD (37). Concomitant with diminished nuclear localization of p65, enhanced expression of p50 was observed in murine TAMs. Nuclear p50 may form heterodimers that contribute to $I l 12 b$ suppression, and in TAMs lacking p50, $I l 12 b$ inducibility was restored (44). IL-10 was shown to impinge on NFkB-dependent signaling by preventing nuclear translocation of p65 in human $(45,46)$ and rat macrophages $(47)$ and of Rel in a mouse macrophage cell line (43). In summary, the protumorigenic and anti-inflammatory effect of ascites may depend on restrainment of NFKB function, and IL-10 may be required for this effect.

We speculated that ascites disables translocation and chromatin binding of $\mathrm{NF \kappa B}$ effector transcription factors and, in consequence, induction of IL12B transcription. To test this hypothesis, the impact of ascites on the induction of $I L 12 B$ by the NFKB transcriptional activators REL and p65 was investigated. Our data show that cell-free OC ascites suppresses IL12B expression by primary human macrophages upon stimulation with LPS and IFN $\gamma$ in vitro via a reversible mechanism. Both REL and p65 translocation to the nucleus was strongly impaired by exposure to ascites. However, induction of CXCL10 mRNA by LPS and IFN $\gamma$ was unaffected in the presence of ascites. High-throughput sequencing approaches after enrichment of several chromatin marks were used to map putative regulatory regions of the REL target genes $I L 12 B$ and CXCL10. In the presence of ascites, REL and p65 were recruited to the $I L 12 B$ and CXCL10 loci, indicating that their reduced nuclear levels are still sufficient for chromatin 
binding. Furthermore, IL-10 contributes to but is not sufficient for full suppression of $I L 12 B$ expression by macrophages in vitro. These data implicate an additional suppressive mechanism, mediated by ascites-borne soluble factors, which acts upstream of $I L 12 B$ transcription.

\section{MATERIALS AND METHODS}

\subsection{Ascites Collection and Isolation of TAMs From Ovarian Cancer Ascites}

Ascites was collected from untreated high-grade serous ovarian carcinoma patients undergoing first-line surgery at the University Hospital Marburg. Informed consent was obtained from all patients according to the protocols approved by the institutional ethics committee. Mononuclear cells were isolated from ascites by Lymphocyte Separation Medium (Capricorn, no. LSM-A) density gradient centrifugation and subsequent enrichment by adherent cell positive selection in autologous ascites. Tumor-associated macrophages were directly harvested for chromatin immunoprecipitation, directly lysed for RNA isolation or cultivated in R5 medium (RPMI 1640 (Life Technologies, no. 61870044) with 5\% (v/v) human $A B$ serum (human serum type AB (male), (Sigma no. H4522)), and $1 \mathrm{mM}$ sodium pyruvate (Sigma, no. S8636)) or in $100 \%$ cell-free autologous ascites for 1-2 days with or without recombinant $50 \mathrm{ng} / \mathrm{ml} \mathrm{IFN \gamma}$ (from E. coli; Biomol, no. 51564) as indicated.

\subsection{Isolation and Culture of Monocyte- Derived Macrophages}

Buffy coats from healthy adult volunteers were kindly provided by the Center for Transfusion Medicine and Hemotherapy at the University Hospital Giessen and Marburg. Mononuclear cells were isolated from peripheral blood mononuclear cells from female healthy donors. Ficoll density gradient centrifugation was performed with Lymphocyte Separation Medium (Capricorn), and the cells were further purified by adherent cell positive selection of healthy donor monocytes. Monocyte-derived macrophages (MDMs) were generated from monocytes (6-12 days differentiation period) from healthy donors by cultivation in RPMI 1640 (Life Technologies, no. 61870044) with 5\% (v/v) human AB serum (human serum type $\mathrm{AB}$ (male), Sigma no. H4522) and $1 \mathrm{mM}$ sodium pyruvate (Sigma, no. S8636) (R5 medium) or in cell-free ascites from ovarian cancer patients, as indicated.

\subsection{Cytokine Treatment}

MDMs were stimulated with $100 \mathrm{ng} / \mathrm{ml}$ LPS (E. coli 0111:b4 L4391; Sigma) and $20 \mathrm{ng} / \mathrm{ml}$ recombinant human IFN $\gamma$ (from $E$. coli; Biomol, no. 51564) or $20 \mathrm{ng} / \mathrm{ml}$ recombinant human IL-10 from HEK293 cells (Biomol, no. 97490) as indicated.

\subsection{RNA Isolation and RT-qPCR}

Total RNA from TAMs was extracted with TRIfast (Peqlab, no. 30-2020) or from MDMs with the NucleoSpin RNA kit (Macherey\&Nagel, no. 740955) according to the manufacturer's instructions. Complementary DNA synthesis was carried out with the iScript cDNA Synthesis Kit (Bio-Rad, no. 170-8891SP) according to the manufacturer's instructions with 250-500 ng of purified RNA per sample. Quantitative PCR analyses were performed in three technical replicates per sample using ABsolute SYBR Green master mix (Thermo Scientific, no. AB-1158B) in Mx3000p and Mx3005 thermocyclers (Stratagene). The ribosomal protein 27, large subunit (RPL27) transcript was chosen for normalization after testing three housekeeping genes selected from our RNA-seq datasets with eight different TAM samples. RT-qPCR was carried out using the following primers: RPL27, AAAGCTGTCATCGTGAAGAAC and GCTGTCACTTTGCGGGGGTAG; IL12B, GCGAGGT TCTAAGCCATTCG and ACTCCTTGTTGTCCCCTCTG; CXCL10, AAGCAGTTAGCAAGGAAAGGTC and GACATA TACTCCATGTAGGGAAGTGA. Raw data were evaluated with the Cy0 method (48) or the MxPro 4.01 software from Stratagene for $\mathrm{Ct}$ value calculation as indicated.

\subsection{IL-12p40 ELISA}

Concentrations of p40 in cell-free supernatants of cultured cells were determined in three technical replicates per sample using an ELISA kit from Biolegend (no. 430706) according to the instructions of the manufacturer.

\subsection{Flow Cytometry Analysis of Macrophages}

MDMs were stained with APC-labeled $\alpha$-CD206 (BioLegend Cat \#321110 RRID:AB_571885) and PE-labeled $\alpha$-CD163 (eBioscience no. 12-1639-42) as described previously (5). Isotype control antibodies were from BD Biosciences, Miltenyi Biotech, and eBioscience. Cells were analyzed by flow cytometry using a FACS Canto II cytometer and FACSDiva software (BD Bioscience), and results were calculated as percentage of positive cells and mean fluorescence intensities (MFI).

\subsection{Subcellular Fractionation}

Subcellular protein fractionation was performed after washing cells twice with ice cold PBS (Sigma). Cell pellets were subsequently lysed in hypotonic cytosol extraction buffer L1 $(5 \mathrm{mM}$ PIPES pH 8.0, $85 \mathrm{mM} \mathrm{KCl,} \mathrm{0.5 \%} \mathrm{(v/v)} \mathrm{NP40,} \mathrm{protease} \mathrm{inhibitor}$ mix (Sigma, no. P8340) 1:1,000) for 20-40 min on ice. Lysates were collected and centrifuged for $5 \mathrm{~min}$ at $2,000 \times g, 4{ }^{\circ} \mathrm{C}$. Cytosolic extract (CE) supernatants were collected, and nuclear pellets were washed once in L1 and subsequently lysed in RIPA buffer (10 mM Tris-HCl pH 7.5, 150 mM NaCl, 1\% NP40 (v/v), $1 \%$ sodium deoxycholate (w/v), 1 mM EDTA), 1:1,000 protease inhibitor Mix (Sigma), $25 \mathrm{U} / \mathrm{ml}$ benzonase (Merck Millipore, no. 70746) for generation of nuclear extracts (NE) in a ratio of 5:1 of cytosol to nuclear extract.

\subsection{Immunoblotting and Protein Quantification}

Immunoblots were performed according to standard protocols using the following antibodies: $\alpha$ - $c$-REL polyclonal antibody (Cell Signaling Technology Cat \#4727 RRID:AB_2178843); $\alpha$-p65/RELA monoclonal antibody (Cell Signaling Technology Cat \#8242 also 8242P, 8242S RRID:AB_10859369); $\alpha-$ LDH polyclonal antibody (Santa Cruz Biotechnology Cat 
\#sc-33781 RRID:AB_2134947); $\alpha$-acetyl-histone H3 polyclonal antibody (Millipore Cat \#06-599 RRID:AB_2115283); $\alpha$ - $\beta$-actin (AC-15) monoclonal antibody (Sigma-Aldrich Cat \#A5441 RRID:AB_476744); $\alpha$-rabbit IgG, HRP-linked (Cell Signaling Technology Cat \#7074 also 7074S, 7074V, 7074P2 RRID:AB_2099233), and $\alpha$-mouse IgG, HRP-linked (Cell Signaling Technology Cat \#7076 also 7076S, 7076V, and 7076P2 RRID:AB_330924). Imaging and quantification was done using the ChemiDoc MP chemoluminescence imaging system and Image Lab software version 5 (Bio-Rad).

\subsection{RNA Interference}

Small interfering RNA transfection was performed according to the manufacturer's protocol using the TransIT-X2 reagent from Mirus (no. 6000) or the Viromer GREEN reagent (Lipocalyx, no. 230055). The following equimolar mixtures of three siRNA oligonucleotides each from Sigma were used for transfection of macrophages: REL SASI-Hs01-00064620, SASI-Hs01-00064621, SASI-Hs01-00064622. Set of four Upgrade ON-TARGETplus from Dharmacon was used for RELA (LU-003533-00-0002), containing four siRNA oligonucleotides. MISSION siRNA Universal Negative Control \#2 from Sigma was used as a control siRNA (si-ctrl). Cells were harvested $48 \mathrm{~h}$ after transfection.

\subsection{Generation of Murine Bone Marrow- Derived Macrophages, II12b Quantitative RT-PCR, and II-12p40 ELISA}

BMDMs were generated by cultivation of $2 \times 10^{6}$ bone marrow cells/well derived from wild-type and $\mathrm{Rel}^{-1-}$ mice in RPMI 1640 medium supplemented with $10 \%$ heat-inactivated FCS and $10 \mathrm{ng} / \mathrm{ml}$ macrophage colony-stimulating factor (PeproTech) in six-well plates. After one week of cell culture, purity was tested by FACS staining for CD11b. Subsequently, BMDMs were stimulated with either LPS alone $(100 \mathrm{ng} / \mathrm{ml})$ or with LPS $(100 \mathrm{ng} / \mathrm{ml})$ in combination with recombinant IFN $\gamma(10 \mathrm{ng} / \mathrm{ml}) .24 \mathrm{~h}$ after stimulation of BMDMs, supernatants from cell cultures were harvested. Murine Il-12p40 was measured with an ELISA kit (BD Biosciences, no. 555165) according to the manufacturer's protocol. Total RNA was extracted from BMDM-derived cell pellets using the High Pure RNA Isolation Kit (Roche, no. 11828665001). cDNA was synthesized using the RevertAid First Strand cDNA Synthesis Kit (Thermo Scientific, no. K1621). Gene expression was analyzed with a 7500 Fast Real-Time PCR System (Applied Biosystems). The gene expression of Hprt1 was measured as an internal control. The following primer sets were used: Hprt1, CTGGTGAAAAGGACCTCTCG and TGAAGTAC TCATTATAGTCAAGGGCA; Ill2b, ATGTGTCCTCAGAAG CTAACCATC and CGTGTCACAGGTGAGGTTCACT.

\subsection{Chromatin Immunoprecipitation and MIRA}

After adherence selection in Greiner tissue culture flasks (no. 660175) in autologous ascites, TAMs were washed with PBS twice. MDMs were differentiated as indicated. Fixation was performed with 1\% formaldehyde in PBS for $10 \mathrm{~min}$ at room temperature followed by quenching with $125 \mathrm{mM}$ glycin for $5 \mathrm{~min}$. Cells were washed with ice-cold PBS twice and harvested using a cell scraper. The pellet was lysed in hypotonic buffer L1 (5 mM PIPES pH 8.0, $85 \mathrm{mM} \mathrm{KCl}$, $0.5 \%(\mathrm{v} / \mathrm{v}) \mathrm{NP} 40$ ) with protease inhibitor mix (Sigma, no. P8340, 1:1,000) for 20-40 min on ice. Nuclei were resuspended in ChIP RIPA buffer (10 mM Tris-HCl pH 7.5, 150 mM NaCl, 1\% NP40 (v/v), $1 \%$ sodium deoxycholate (w/v), 1 mM EDTA) supplemented with 1:1,000 protease inhibitor mix (Sigma), incubated on ice for 10-20 min and sheared with a Branson S250D Sonifier (Branson Ultrasonics) using a microtip in $1 \mathrm{ml}$ aliquots in $15 \mathrm{ml}$ conical tubes. 52 pulses of $1 \mathrm{~s}, 4$ s pause, $20 \%$ amplitude were applied with cooling of the sample in an ice-ethanol mixture or in a $15 \mathrm{ml}$ tube cooler (Active Motif, no. 53077). A 15 min 20,000×g supernatant was precleared with $10 \mu \mathrm{g}$ of IgG coupled to $100 \mu \mathrm{l}$ of blocked sepharose slurry (see below) for $45 \mathrm{~min}$ at $4{ }^{\circ} \mathrm{C}$ with agitation. IP was carried out with $300 \mu \mathrm{l}$ of precleared chromatin, equivalent to $3-8 \times 10^{6}$ cells. For MIRA, an aliquot of the sample was reverted as described below, purified on a Qiagen PCR purification column (no. 28106), and $250 \mathrm{ng}$ of DNA were used according to the manufacturer's instructions (Methylcollector Ultra, Active Motif, no. 55005). ChIP was performed and evaluated as described using $4 \mu \mathrm{g}$ per sample of the following antibodies: IgG pool, (Sigma-Aldrich Cat \#I5006 RRID:AB_1163659) or normal rabbit IgG (Cell Signaling Technology Cat \#2729S RRID:AB_1031062); $\alpha$-c-REL (Santa Cruz Biotechnology Cat \#sc-70 RRID:AB_2178727 and Santa Cruz Biotechnology Cat \#sc-71 RRID:AB_2253705, 1:1 mixture); $\alpha$-p65/RELA (Diagenode Cat \#C15310256 RRID:AB_2721009); $\alpha$-p65/RELA (Cell Signaling Technology Cat \#8242 also 8242P, 8242S RRID:AB_10859369); $\alpha$-H3K27me3 (Diagenode Cat \#pAb069-050 also ENCAB000ARJ RRID:AB_2616049); $\alpha$-H3K4me3 (Diagenode Cat \#pAb-003-050 also ENCAB000BKU, C1541000350, C15410003-10 RRID:AB_2616052); $\alpha$-H3K27ac (Diagenode, Diagenode, Cat \#C15410174, RRID:AB_2716835); $\alpha$-H3K4me1 (Diagenode Cat \#pAb-037-050, RRID:AB_2561054); $\alpha$-H3K9me3 (Diagenode Cat \#pAb-056-050, RRID:AB_2616051); $\alpha$-H3K36me3 (Abcam Cat \#ab9050, RRID:AB_306966); $\alpha$-C/EBP $\beta$ (Santa Cruz Biotechnology Cat \#sc-150, RRID:AB_2260363). For precipitation, a mixture of protein A and protein G sepharose (GE Healthcare life sciences, no. 1752800 and no. 1706180) was washed twice with ChIP RIPA buffer and blocked with $1 \mathrm{~g} / \mathrm{l} \mathrm{BSA}$ and $0.4 \mathrm{~g} / \mathrm{l}$ sonicated salmon sperm DNA (Life Technologies no. 15632011) overnight. $50 \mu$ l of blocked bead slurry (1:1 volume ratio with liquid phase) were used per IP. Samples were washed once in buffer I (20 mM Tris pH 8.1; 150 mM NaCl; 1\% (v/v) Triton X-100; 0.1\% (w/v) SDS; 2 mM EDTA), once in buffer II (20 mM Tris pH 8.1; $500 \mathrm{mM} \mathrm{NaCl} ; 1 \%$ (v/v) Triton X-100; 0.1\% (w/v) SDS; 2 mM EDTA), twice in buffer III (10 mM Tris pH 8.1; $250 \mathrm{mM} \mathrm{LiCl;} 1 \%(\mathrm{v} / \mathrm{v})$ NP40; 1\% (w/v) sodium deoxycholate; $1 \mathrm{mM}$ EDTA) on ice, and twice in Qiagen buffer EB (no. 19086) at room temperature. Immune complexes were eluted twice with $100 \mathrm{mM} \mathrm{NaHCO}_{3}$ and $1 \%$ SDS (w/v) under agitation. Eluates were incubated overnight at $65{ }^{\circ} \mathrm{C}$ after adding $10 \mu \mathrm{g}$ of RNase A and $20 \mu \mathrm{g}$ of proteinase $\mathrm{K}$ in the presence of $180 \mathrm{mM} \mathrm{NaCl}, 35 \mathrm{mM}$ Tris- $\mathrm{HCl} \mathrm{pH} \mathrm{6.8,} \mathrm{and} 9$ mM EDTA. An input sample representing $1 \%$ of the chromatin used per IP was reverted in parallel. Samples were purified using the Qiagen PCR purification kit according to the manufacturer's instructions, except that DNA-binding lipids were removed by washing the matrix twice with pure methanol as described (49) previous to the final washing step with the buffer included in the kit. ChIP-qPCR was performed in three technical replicates per sample with the ABsolute SYBR 
Green master mix (Thermo Scientific, no. AB-1158B) in Mx3000p and Mx3005 thermocyclers (Stratagene) using the following primers: $I L 12 B-1,200$ bp CCATCCCTGCTCTCGACCT and GAAATCTGCGCCCGCCTAAA; IL12B TSS, AGTGCTTA CCTTGCTCTGGG and TACCAGCAACAGCAGCAGAA; IL12B +20 kbp, ACGCCGCCCTAGAAGAAG and TCCC TTTCACCTTCTCTGGA; CXCL10 -5,000 bp, AGCTGGTG CAGAATATGCCTT and CACTGTGAGCTCGGGGAATC; CXCL10 TSS, GAAGTCCCATGTTGCAGACTC and AACAGT TCATGTTTTGGAAAGTGA. Data were evaluated using the MxPro 4.01 software from Stratagene for Ct value calculation. Relative recoveries were determined as percentage of input using a $\Delta$ Ct method (50).

\subsection{Library Preparation and High- Throughput Sequencing}

Libraries were synthesized from 1-2 ng of genomic DNA using the MicroPlex kit (Diagenode, no. C05010011) according to the manufacturer's instructions. Samples were sequenced on an Illumina Hi-Seq 1000 (single-ended, 50 bp).

\subsection{Statistical Tests}

Paired $t$-tests were used to calculate $p$-values.

\subsection{Bioinformatics and Data Deposition}

Mapping of ChIP-Seq reads and peak calling were carried out as described (49). Peaks were filtered for at least 30 deduplicated tags and a fold change (FC) over IgG of $\geq 2$ (normalized total read counts). All genomic sequence and gene annotation data were retrieved from Ensembl revision 74. ChIP-seq data were deposited at ArrayExpress (no. E-MTAB-6297).

\section{RESULTS}

\subsection{Suppression of IL12B in Monocytic Cells From Ovarian Carcinoma Patients Is Mediated by Soluble Factors From Ascites}

We initially assessed whether the inability of TAMs to produce IL-12p40 is reversible in our experimental setups. Ex vivo TAMs were in autologous ascites or in normal medium (RPMI 1640 supplemented with $5 \%$ adult human serum, "R5") for 1 day or 2 days and stimulated with LPS and IFN $\gamma 24 \mathrm{~h}$ prior to harvesting. IL12B expression was induced on both mRNA (Figure 1A; RT-qPCR) and protein levels (Figure 1B; $\alpha$-p40 ELISA) in cells from four out of eight patients cultivated in ascites. After cultivation in autologous ascites for 2 days, the transcript and the protein were inducible to a lesser extent; LPS and IFN $\gamma$ were added to the cultures $24 \mathrm{~h}$ prior to harvesting. The presence of IFN $\gamma$ for the whole time of cultivation in 2 days samples led to increased induction. We conclude that suppression of $I L 12 B$ in TAMs happens primarily at the transcriptional level, and suppression can be counteracted by IFN $\gamma$, as it was shown by others (34). Furthermore, we found that expression was inducible without IFN $\gamma$ pretreatment in some samples (Figure 1). It is important to note that we added IFN $\gamma$ in parallel with LPS to non-pretreated cultures, while the aforementioned study used LPS as a single stimulus after pretreatment with IFN $\gamma$ (34). Apparently either a subpopulation of the cells from these patients is responsive to LPS and IFN $\gamma$ ex vivo, or the autologous ascites samples are less capable of $I L 12 B$ suppression. Strikingly, TAMs cultivated in normal medium in the absence of IFN $\gamma$ were able to induce IL12 transcription to similar levels as TAMs cultivated in ascites in the presence of IFN $\gamma$ (Figure 1A). Cultivation in normal medium led to detectable transcripts in all donors analyzed $(N=8)$, while cultivation in the presence of IFN $\gamma$ had this effect only in five out of eight donors. Taken together, this suggests that ascites-mediated suppression of $I L 12 B$ induction is reversible, and this may depend on differences between ascites samples as well as cells from individual donors. Presumably due to biological heterogeneity, differences between the induced and non-induced conditions regarding p40 protein levels were not statistically significant when analyzing supernatants from TAMs cultivated in ascites (Figure 1B).

IL12B expression data from TAMs cultivated in normal medium or in ascites supplemented with IFN $\gamma$ suggest that these cells are generally capable of inducing this transcript. Its induction in the presence of ascites could be due to an effect on monocytic cells that were freshly recruited to the tumor microenvironment and thus not yet strongly affected by ascites, or the effect of ascites on $I L 12 B$ could be of a reversible nature. We, therefore, conducted the following experiments in MDMs differentiated from healthy donor monocytes in vitro in normal medium (R5) or in ascites as described previously (7) — this culture system allows for controlled differentiation as well as short-term treatment of cell populations that are less heterogeneous than TAM isolates. High levels of IL-12p40 can be produced by MDMs, and this is suppressed by ascites but overcome in the presence of $\operatorname{IFN} \gamma(7,34)$. Priming of MDMs differentiated in $\mathrm{R} 5$ with IFN $\gamma$ prior to stimulation with LPS and IFN $\gamma$ did not potentiate IL-12p40 production (Figure S1 in Supplementary Material), indicating that our culture conditions do not limit expression of p40 in this regard.

\subsection{Suppression of IL12B Can Be Reversible Upon Ascites Withdrawal}

When MDM differentiation was carried out in normal medium for 6 days and followed by exposure to ascites for 1 day (short-term exposure, which was applied simultaneously with LPS and IFN $\gamma$ ), IL12B (Figure 2A, $N=5$ ), and IL-12p40 (Figure 2B, $N=8$ ) induction was efficiently suppressed. The effect was reverted in all MDM samples on RNA level by ascites withdrawal for 1 day after short-term exposure (Figure 2A), which was statistically significant; however, cells from most donors produced little or no p40, while others produced considerable levels (3/8; Figure 2B). This further point to a highly variable effect due to heterogeneity between biological samples, which could be due to the use of different ascites samples, different healthy donors, or both.

To clarify whether long-term exposure to ascites has lasting effects on the capacity of MDMs to express $I L 12 B$, monocytes from three healthy donors were differentiated either in R5 or in each of five different cell-free ascites samples from OC patients for 12 days (long-term exposure) in order to mimick the cellular state after differentiation in the ovarian tumor microenvironment. $I L 12 B$ induction by LPS and IFN $\gamma$ was markedly reduced 

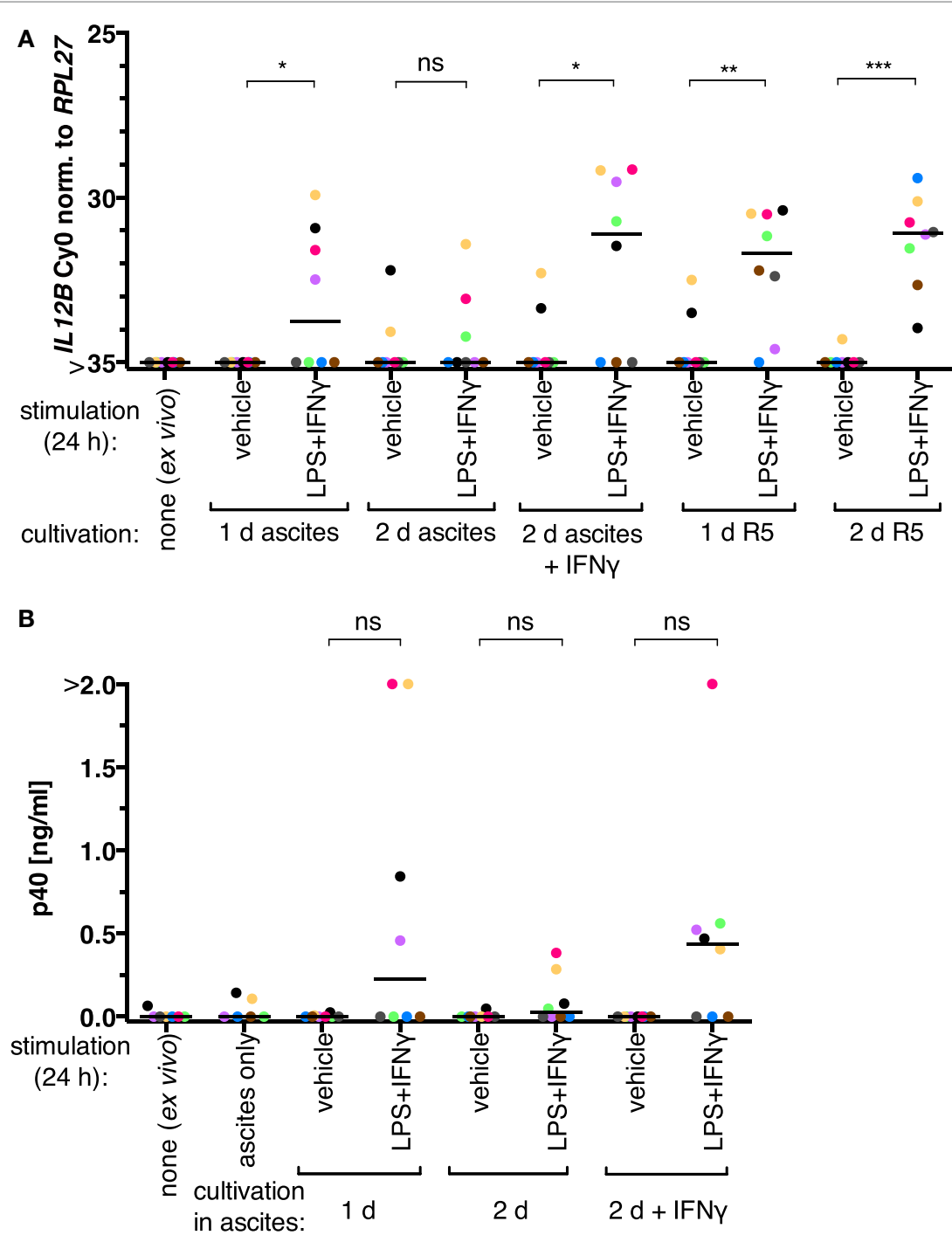

FIGURE 1 | IL-12 production by tumor-associated macrophages from ovarian carcinoma patients is suppressed by ascites in vitro. (A) Ex vivo TAMs ( $N=8)$ were cultivated in autologous ascites in the presence or in the absence of IFN $\gamma$ or in normal medium (R5) as indicated, and non-cultivated ex vivo TAMs served as an additional control. LPS and IFN $\gamma$ or their respective solvents (vehicle) were added to the culture supernatants for $24 \mathrm{~h}$ prior to harvesting, and IL $12 B$ expression was measured by RT-qPCR. (B) Secreted IL-12p40 from these TAMs was measured by ELISA ( $N \geq 6$ ). Cell-free autologous ascites was used as additional control. Each dot denotes a biological replicate. Median values are indicated by horizontal bars. Colors encode individual patients, and colors are consistent between panels within this figure. Statistical significances were calculated with paired $t$-tests. ${ }^{\star} P<0.05$; ${ }^{\star \star} P<0.01$; ${ }^{\star \star} P<0.001$; ns, not significant.

in ascites-differentiated MDMs vs. MDMs differentiated in R5 medium on both mRNA (Figure 2C) and protein levels (Figure 2D). Although, suppression was not complete in some cultures, and this depended on both the donor cells and the ascites samples. MDMs differentiated in ascites for 6 days clearly induced $I L 12 B$ expression after ascites withdrawal for 6 days (Figures 2C,D), albeit to lower levels than MDMs cultivated in normal medium for 12 days. Importantly, this happened in MDM cultures from all three donors in combinations with different ascites samples; $\mathrm{p} 40$ was detected in all but two combinations of donor cells with ascites samples. Induction after ascites withdrawal was elevated, but the effect did not reach statistical significance except for one comparison (Figure 2D, upper right panel). This can be attributed to the limiting number of samples analyzed and, importantly, to biological variation. Some ascites samples exerted suppressive effects that resulted in no or lesser reversibility, and MDMs from individual donors were susceptible to suppression by different ascites samples in a differential manner. We conclude that suppression of $I L 12 B$ expression by OC ascites is reversible by ascites withdrawal in principle. On RNA level, reversal was more effective after short-term exposure. Since suppression was functional when ascites was added simultaneously with LPS and IFN $\gamma$, these data suggest that a suppressive mechanism acts immediately at the level of transcription and can affect MDMs which were differentiated in the absence of ascites (Figure 2A). Additional suppressive mechanisms apparently act posttranscriptionally (Figure 2B). 

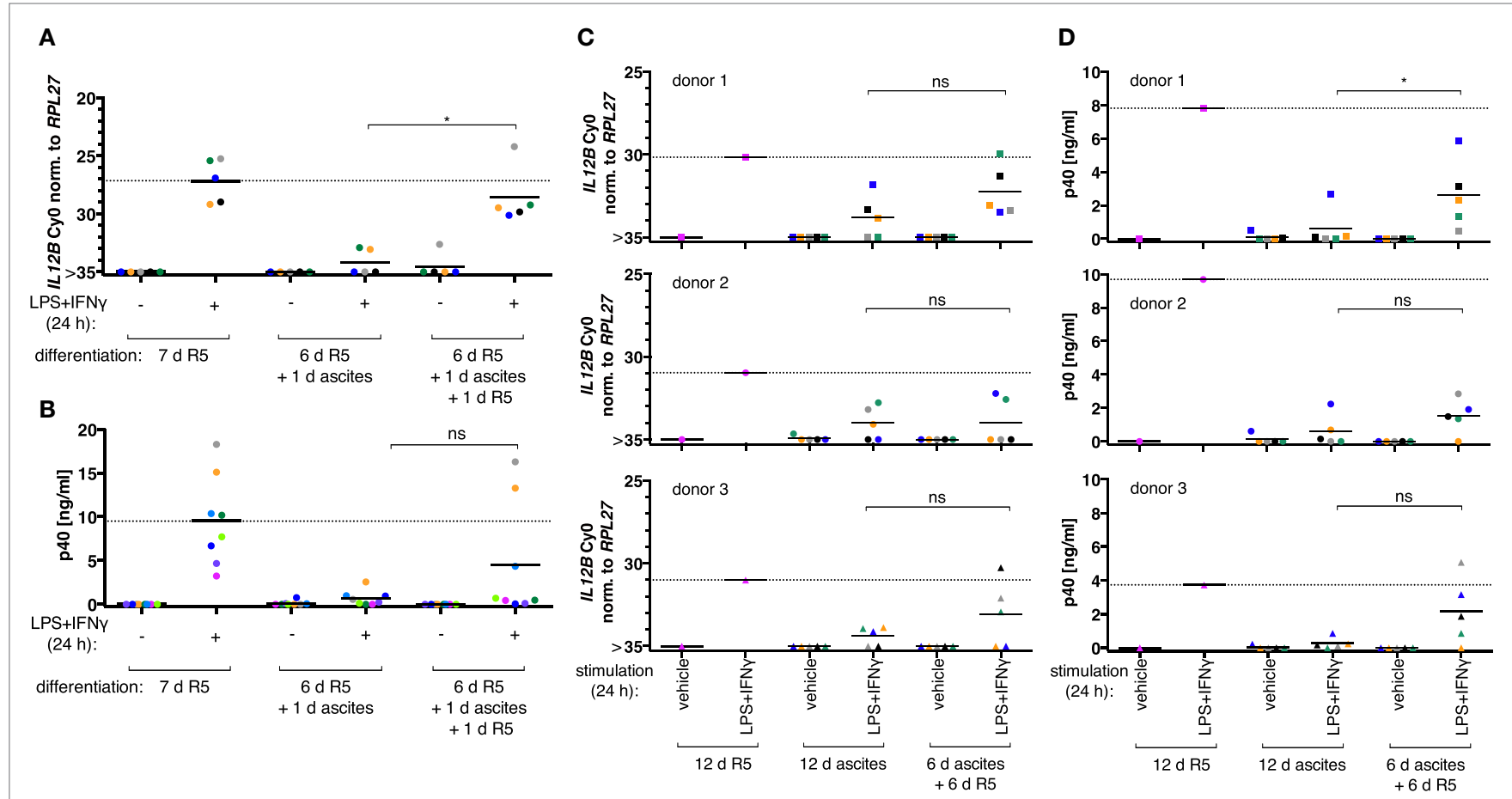

FIGURE 2 | Ascites-mediated suppression of IL12B in monocyte-derived macrophages is reversible upon ascites withdrawal. IL12B expression induced by LPS and IFN $\gamma$ in MDMs (differentiated as indicated) was measured by RT-qPCR (A,C) or in culture supernatants by ELISA (B,D). Cells were stimulated for $24 \mathrm{~h}$ prior to harvesting, or the respective solvents (vehicle) were added, respectively. (A,B) Each dot denotes a biological replicate (healthy donor; $N \geq 5$ ) randomly combined with different ascites samples encoded by color. $(\mathbf{C}, \mathbf{D})$ Colors encode ascites samples from five different patients; shapes encode different healthy donors $(N=3)$. Median values are indicated by horizontal bars. Statistical significances were calculated with paired $t$-tests. ${ }^{\star} P<0.05$; ns, not significant.

The observation that $I L 12 B$ suppression by ascites is rapid and reversible suggests that at least some of the mechanisms involved do not elicit a stable macrophage polarization state. In order to systematically characterize the state of MDMs upon suppression of IL $12 B$ expression by ascites in vitro, monocytes were differentiated in normal medium, in ascites in the presence of recombinant IFN $\gamma$, or in normal medium for 6 days followed by ascites for 1 day (short-term exposure). Under these conditions, we found that ascites leads to increased expression of the markers CD163 and CD206, indicating alternative polarization (Figure S3 in Supplementary Material). Upon cultivation in ascites in the presence of IFN $\gamma$, induction of these markers was partially reversed, concomitant with a restoration of $I L 12 B$ inducibility. It can, therefore, not be excluded that an altered macrophage differentiation state might contribute to the blockade of $I L 12 B$ transcription in TAMs. This notion would be consistent with the observed stable IL12B suppression by a prolonged exposure of MDMs to ascites.

\subsection{Ascites Reversibly Suppresses Nuclear Translocation of REL and p65}

Since suppression of $I L 12 B$ induction by ascites is immediate and acts on the level of transcription, an obvious assumption is that NFKB function is compromised due to high levels of IL-10 in ascites. Nuclear translocation of REL was detected after both 1 and $2.5 \mathrm{~h}$ of stimulation with LPS and IFN $\gamma$ in our MDM culture system in cells differentiated in normal medium (Figure
S2B in Supplementary Material), and the latter time point coincided with measurable synthesis of IL12B mRNA (Figure S2A in Supplementary Material). This is in line with the regulation of "second wave" NFKB target genes such as IL12B (51). For subsequent analyses, LPS and IFN $\gamma$ were added to the culture supernatants $2.5 \mathrm{~h}$ prior to harvesting.

According to our mass spectrometry data, REL, p65, and p50 are the main NFKB transcription factors expressed in TAMs (52). Nuclear translocation of the transcriptional activators REL and p65 was assessed by subcellular fractionation of MDMs after short-term ( $24 \mathrm{~h}$ ) or long-term exposure to ascites (differentiation for 6 days or more). Representative immunoblots are shown in Figures S4B,C in Supplementary Material. Long-term exposure abrogated measurable REL translocation in six out of nine donors (Figure 3A) and translocation of p65 in all three donors analyzed (Figure 3C). Short-term exposure was less effective; detectable REL translocation was lost in two out of six donors (Figure 3B) and that of p65 in one out of three (Figure 3D). Although, exposure to ascites strongly reduced nuclear translocation in cells from all donors. Strikingly, ascites withdrawal for 1 day reinstalled nuclear localization of both REL (Figure 3B) and p65 (Figure 3D) in MDMs exposed to ascites for $24 \mathrm{~h}$, which, however, did not reach the same levels as those of MDMs not exposed to ascites. In cells from the same donors used for short-term exposure experiments, reinstallation happened only in two out of three donors for REL (Figure 3A) and in one out of three donors for p65 (Figure 3C) after long-term exposure and 6 days of ascites withdrawal. Taken 

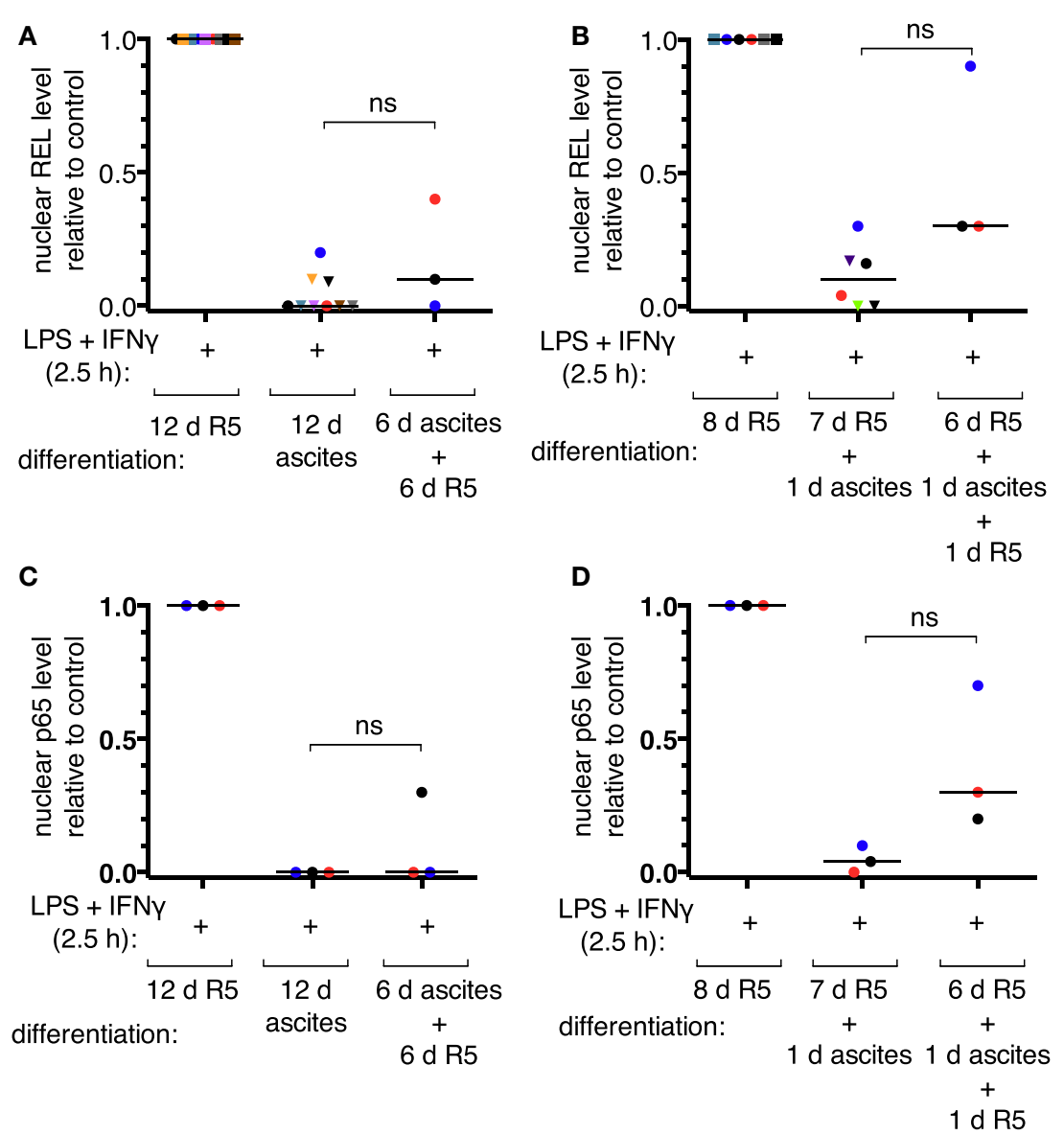

FIGURE 3 | Ascites impairs inducible nuclear translocation of REL and p65 in monocyte-derived macrophages. MDMs were differentiated in R5 medium or in ascites and cultivated consecutively as indicated. Cells were incubated with LPS and IFN $\gamma$ for $2.5 \mathrm{~h}$ or their respective solvents (vehicle), harvested, and subcellular fractionation was performed. After immunoblotting, chemoluminescence was measured, nuclear levels of REL were calculated relative to the R5 control population after long-term ascites exposure with $(N=9)$ or without $(N=3)$ ascites withdrawal $(\mathbf{A})$ or after short-term exposure $(\mathbf{B})$, respectively $(N=6$ or $N=3$ as plotted). Nuclear p65 levels were analyzed in a subset of the same samples $(N=3)$ accordingly $(\mathbf{C}, \mathbf{D})$. Each color denotes a biological replicate (combination of healthy donor and randomly chosen ascites sample). Dots mark samples which were probed for both REL and p65 nuclear translocation. The code is consistent between panels within this figure. Median values are indicated by horizontal bars. Statistical significances were calculated with paired $t$-tests; ns, not significant $(P>0.05)$.

together, this demonstrates that impairment of REL and p65 nuclear translocation by ascites is rapid, and the effect can be reversible upon ascites withdrawal.

Nuclear localization of the NFKB transcriptional activators is controlled by I $\kappa \mathrm{B}$ proteins, and their involvement in IL-10mediated impairment of $\mathrm{NF \kappa B}$ function is well documented

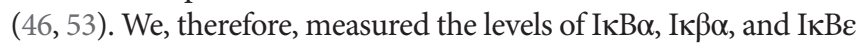
proteins after stimulation of MDMs with LPS and IFN $\gamma$ (Figures S5A-C in Supplementary Material; representative immunoblots are shown in Figure S4A in Supplementary Material) and found that, despite nuclear localization of REL, cellular I $\mathrm{B} \alpha$ levels were increased upon stimulation in all cell populations analyzed (Figure S5A in Supplementary Material). The levels of I $\mathrm{B} \beta$ and $\mathrm{I} \kappa \mathrm{B} \varepsilon$ were strongly decreased upon stimulation under all conditions (Figures S5B,C in Supplementary Material). This is in line with the described positive feedback mechanism of $\mathrm{I \kappa B} \alpha$ after its initial rapid degradation, which subsequently leads to enhanced protein levels (53). Inducible Ік $\beta \alpha$ degradation was more pronounced in cells differentiated in ascites (Figure S5B in Supplementary Material). In conclusion, we did not observe a correlation between the levels of I $\mathrm{B}$ p proteins and the diminished capacity of $I L 12 B$ transcription in MDMs exposed to ascites relative to non-exposed cells.

\subsection{Induction of CXCL10 Expression Is Not Prevented in the Presence of Ascites}

Because nuclear translocation of REL and p65 is diminished in the presence of ascites, we speculated that transcription of target genes other than IL12B may be affected. CXCL10/IP10 is a bona fide REL (54-56) and p65 target gene (57) which, however, is expressed in TAMs (7). A subset of cDNA samples shown in Figure 2 was used to test ascites-mediated effects on the induction of CXCL10 expression. We found that the levels of this highly inducible transcript $-\Delta \mathrm{Cy0} \geq 10$ in most sample combinations, which is equivalent to $>1,000$-fold induction-were weakly reduced after 
short-term exposure, reaching statistical significance, and significantly elevated after short-term exposure and ascites withdrawal (Figure S6A in Supplementary Material). However, mean CXCL10 levels (calculated for a total of six samples from three individual donors) were unaffected in MDMs stimulated with LPS and IFN $\gamma$ after long-term exposure and ascites withdrawal (Figure S6B in Supplementary Material). CXCL10 expression was uniformly high upon induction after both short-term and long-term exposure to ascites (Figures S6A,B in Supplementary Material). In some MDM populations, transcript levels were inducible to even higher levels compared to the control sample after long-term exposure (Figure S6B in Supplementary Material). In summary, this argues against a direct effect of ascites on CXCL10 transcription. Therefore, our data do not show that mRNA synthesis of CXCL10 is prevented in the presence of ascites. This argues against a general ascites-dependent perturbation of the function of REL and $\mathrm{p} 65$ provided that CXCL10 is a direct target gene.

\subsection{Chromatin Marks and Regulatory Elements at the IL12B Locus}

Since translocation of REL and p65 is impaired in the presence of ascites, and $I L 12 B$ mRNA induction is prevented, while that of CXCL10 is not, an obvious hypothesis is that these transcripts are subject to gene-specific regulation. In order to address ascitesmediated effects on NFkB target genes, which are possibly locusspecific, we sought to map histone modifications and regulatory elements at these REL and 65 target genes in primary macrophages in an unbiased approach. To this end, ChIP-seq was performed in ex vivo OC TAMs with antibodies against the histone modification marks H3K4me1 (histone $\mathrm{H} 3$ lysine 4 monomethylation), H3K4me3, H3K9me3, H3K27me3, H3K27ac (H3K27 acetylation), $\mathrm{H} 3 \mathrm{~K} 36 \mathrm{me} 3$, as well as the transcription factor $\mathrm{C} / \mathrm{EBP} \beta$. Genome browser snapshots of the IL12B locus (Figure 4A) indicate that H3K4me1, which marks enhancer sequences, was detected at four sequence stretches within $25 \mathrm{kbp}$ from either end of the coding region. These stretches are distinguished by the following features: (I) $\mathrm{C} / \mathrm{EBP} \beta$ binds to a region $11 \mathrm{kbp}$ upstream of the IL12B transcription start site (TSS); (II) a region $7 \mathrm{kbp}$ upstream of the TSS is decorated with $\mathrm{H} 3 \mathrm{~K} 27 \mathrm{ac}$, which marks active positive regulatory elements; (III) a local enrichment of $\mathrm{H} 3 \mathrm{~K} 4 \mathrm{me} 3$ is localized 1,200 bp upstream of but not at the TSS itself, where this mark is usually found at active and poised genes (58); (IV) a region $4,500 \mathrm{bp}$ downstream of the gene $(20 \mathrm{kbp}$ downstream of the TSS) harbors H3K27me3-modified nucleosomes, indicative of the Polycomb repressive complex 2 (58).

All four designated regions harbor highly conserved sequences, and none of them carry the heterochromatin mark $\mathrm{H} 3 \mathrm{~K} 9 \mathrm{me} 3$. Consistent with minute or absent expression of IL12B transcripts in ex vivo OC TAMs (7), the coding region is devoid of the transcription elongation marker H3K36me3. Poorly annotated transcripts originate from regions III and IV. These long non-coding RNAs generally correlate with regulatory roles of the respective DNA sequences (59). Region II possibly is in spatial proximity to region $\mathrm{I}$, since a minor but noticeable enrichment was caused by the $\mathrm{C} / \mathrm{EBP} \beta$ antibody at this site, which could be due to indirect crosslinking. We assume that regions I and II do not mediate repression due to the presence of the activating $\mathrm{H} 3 \mathrm{~K} 27 \mathrm{ac}$ mark at region II and the absence of repressive marks at both regions.

The unusual configuration of the H3K4me3 mark appearing at a $-1,200$ bp upstream site (region III) but not at the TSS seems to be a hitherto undescribed characteristic of a set of cytokineencoding genes: in our dataset, we observed similar distances at, for instance, CXCL10/IP10 (-5,000 bp) and IL2 (-2,000 bp), which both are REL target genes (42, 54-56, 60). Genome browser snapshots of both loci are shown in Figures S9A,B in Supplementary Material. CXCL10 is expressed in OC TAMs as well as in peritoneal macrophages from tumor-free patients $(6,7)$, while IL2 is not a transcribed gene in macrophages and hence is devoid of active marks but apparently harbors H3K4me3- and H3K4me1-modified nucleosomes within 2,000 bp of its TSS. We excluded the possibility that these $\mathrm{H} 3 \mathrm{~K} 4 \mathrm{me} 3$ upstream shifts relative to the TSSs are due to mapping artifacts; moreover, these observations were confirmed in published datasets such as those from ENCODE (ChIP-seq tracks for H3K4me3; GSM1003536 and GSM945225 for monocytes; and GSM788075 for PBMCs) and an early ChIP-seq study in the Jurkat human T cell line (61).

Strikingly, a weak enrichment of region IV was also caused by the H3K4me3 antibody, which strongly enriches region III. Reciprocally, reads from the $\alpha-\mathrm{H} 3 \mathrm{~K} 27 \mathrm{me} 3 \mathrm{ChIP}$, which are most prominent at region IV, are more densely spaced in an extended stretch, which encompasses region III and the TSS of $I L 12 B$, relative to the coding region. Taken together, region IV may be in proximity to region III in OC TAMs, since each region's more prominent of these two histone marks is, to a lesser extent, mirrored at the other region, presumably due to indirect crosslinking. The presence of both H3K4me3 and H3K27me3 at the same locus is reminiscent of the bivalent state which allows for stable repression and comparably fast induction of expression that was first described for developmental genes (58); however, bivalent genes carry the marks at or close to their TSSs.

To investigate whether cytosine methylation is involved in repression of $I L 12 B$ in TAMs, we used MIRA (methylated CpG island recovery assay)-seq (62). CpG island methylation was not detected at the promoter region of $I L 12 B$, while robust signals originated at the penultimate exon and a region $9 \mathrm{kbp}$ upstream of the TSS (Figure 4A). Importantly, the promoter as well as conserved sequences upstream (region I) and downstream of the coding region, $20 \mathrm{kbp}$ from the TSS (region IV), harbor CpG islands according to DBCAT analysis (63), but were not enriched by MIRA. The human Jurkat $\mathrm{T}$ cell line and other cell lines analyzed show an enrichment of methylated $\mathrm{CpG}$ sequences at the promoter and region IV according to ENCODE datasets (with GEO accession numbers; Jurkat: GSM999367, HeLa-S3: GSM999337, H1: GSM999379, HepG2: GSM999338, HL-60: GSM999386, GM12878: GSM999376, K562: GSM999341, HUVEC: GSM999364, Ovcar-3: GSM999393). This potentially means that methylation of region IV and the promoter regulates cell-type specific expression of IL12B.

In summary, the next-generation sequencing analyses led to the hypothesis that suppression of $I L 12 B$ in TAMs is mediated by an $\mathrm{H} 3 \mathrm{~K} 27 \mathrm{me} 3$-dependent mechanism and involves the promoter/-1,200 bp region and a putative silencer element 
A

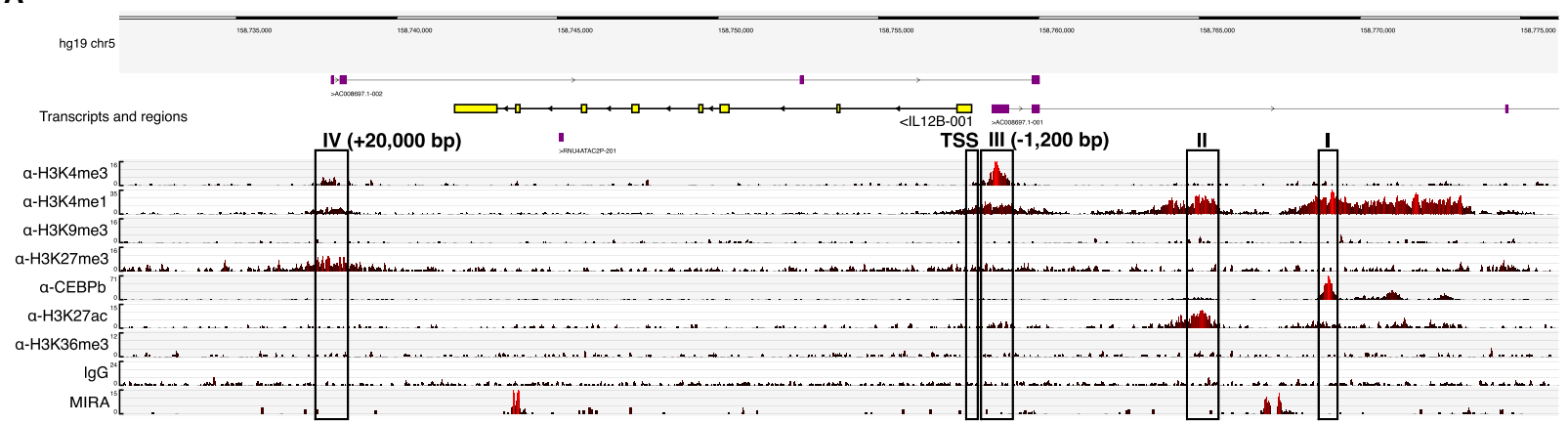

B $\quad I L 12 B-1,200$ bp E

IL 12B -1,200 bp (MDMs) (region III)

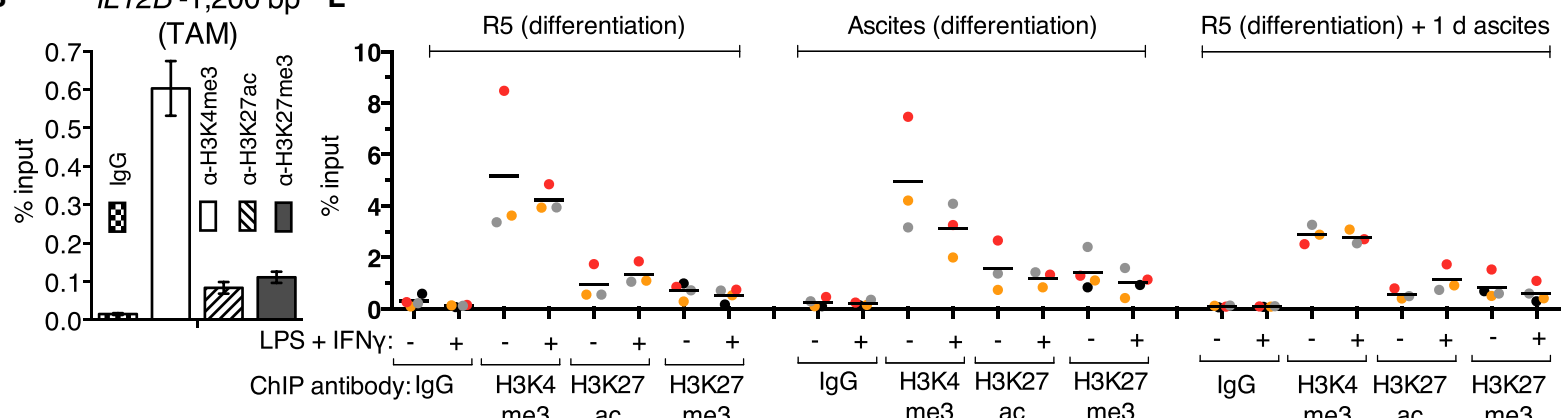

C

IL12B TSS

F R5 (differentiation)

IL12B TSS (MDMs)

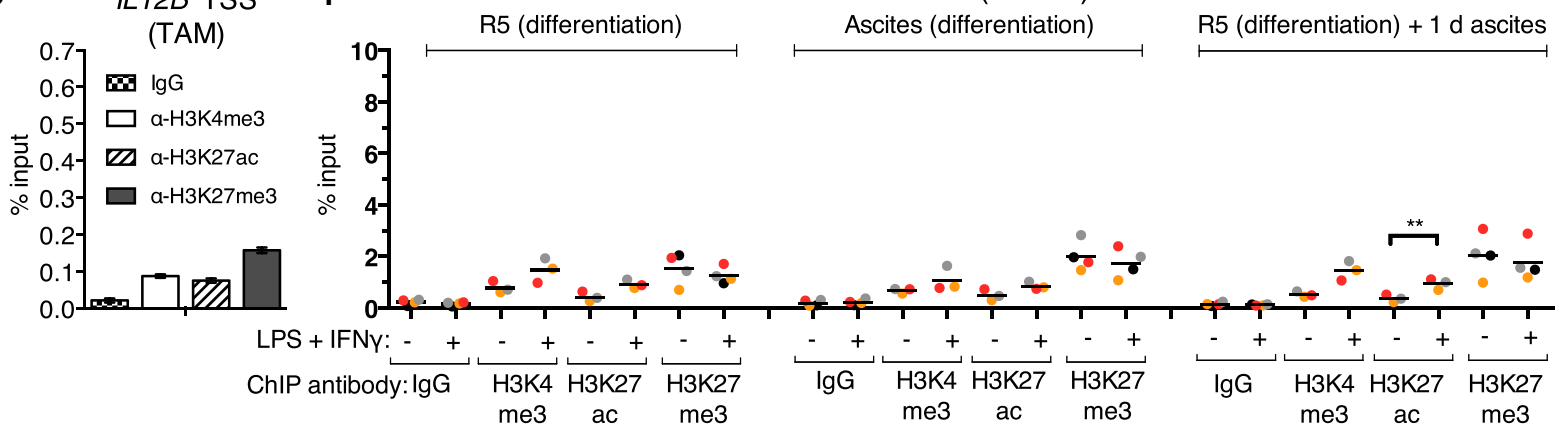

D

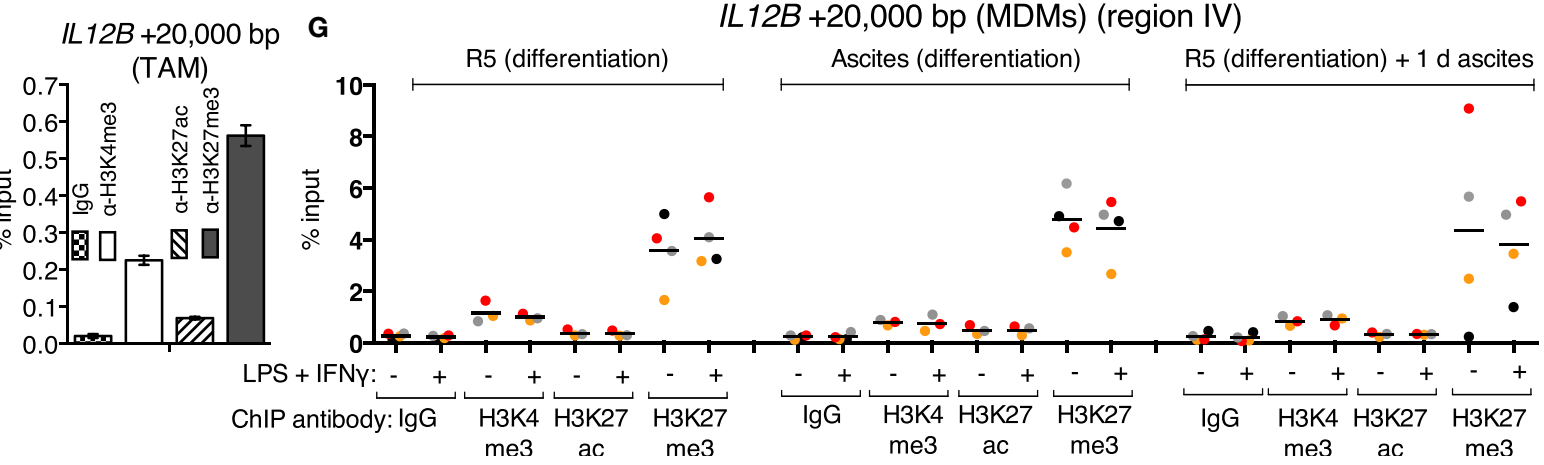

FIGURE 4 | Chromatin marks at the IL12B locus. (A) A genome browser snapshot, including ChIP-seq tracks for $\alpha-H 3 K 4 m e 3, \alpha-H 3 K 4 m e 1, \alpha-H 3 K 9 m e 3$, $\alpha-H 3 K 27 m e 3, \alpha-C / E B P \beta, \alpha-H 3 K 27 a c, \alpha-H 3 K 36 m e 3$, IgG (unspecific polyclonal rabbit lgG pool) chromatin immunoprecipitations, and MIRA from a TAM sample freshly isolated from ovarian carcinoma ascites. Regions of interest are highlighted by rectangles. (B-D) ChIP-qPCR analyses of the indicated histone marks in an independent TAM sample at the indicated genomic locations amplified by specific primers are indicated. The error bars denote SDs from technical PCR replicates. (E-G) ChIP-qPCR analyses of the indicated histone marks in MDMs from three different pooled donor populations ( $N=3 ; N=4$ for lgG and $\alpha$-H3K27me3 samples) differentiated in normal medium, in ascites, or in normal medium followed by ascites for 1 day. Cells were stimulated with or without LPS and IFNy (+) or their respective solvents (-) $2.5 \mathrm{~h}$ prior to harvesting. Genomic regions were amplified by specific primers are indicated. Each dot denotes a biological replicate; for each replicate, MDMs from six donors were pooled after harvesting of the cells for each experiment. Median values are indicated by horizontal bars. Colors encode ascites samples from individual patients, and colors are consistent between panels within this figure, Figure $\mathbf{5}$ and Figure S9 in Supplementary Material. Statistical significances were calculated with paired $t$-tests. ${ }^{*} P<0.01$; all other analyses did not show significance $(P>0.05)$. 
$20 \mathrm{kbp}$ downstream of the TSS. For further analyses, we focused on elements that harbor repressive marks (regions III: $-1,200 \mathrm{bp}$ and IV: $+20 \mathrm{kbp}$ ) as well as the TSS. The observed enrichment of $\mathrm{H} 3 \mathrm{~K} 4 \mathrm{me} 3$ and $\mathrm{H} 3 \mathrm{~K} 27 \mathrm{me} 3$ at these elements was confirmed by ChIP-qPCR in a different TAM sample (Figures 4B-D).

Using ChIP-qPCR, analysis of the chromatin marks H3K4me3, $\mathrm{H} 3 \mathrm{~K} 27 \mathrm{ac}$, and $\mathrm{H} 3 \mathrm{~K} 27 \mathrm{me} 3$ was performed in MDMs differentiated in normal medium, in ascites, or in normal medium followed by exposure to ascites for one day in order to measure possible changes upon short-term and long-term suppression (Figures 4E-G). Cells were stimulated with LPS and IFN $\gamma 2.5 \mathrm{~h}$ prior to fixation; at this time point, REL and p65 were detected in the nucleus (Figure 3; Figure S2B in Supplementary Material), and IL12B transcripts were detectable shortly thereafter (Figure S2A in Supplementary Material). The observed relative levels of the analyzed histone modifications generally mirrored the observations made in TAMs (Figures 4B-D), with absolute recoveries in MDMs being higher due to lower cell numbers that were obtained from MDM cultures in comparison to TAMs from large volumes of ascites. H3 lysine 4 trimethylation levels did not change consistently; at the TSS, levels were low but uniformly increased upon stimulation with LPS and IFN $\gamma$. At the IL12B TSS, the H3 lysine 27 acetylation signal increased consistently (Figure 4F) upon stimulation, and this was statistically significant in the short-term ascites-exposed population. Mean H3K27me3 signals at the TSS of IL12B were slightly elevated in MDMs exposed to ascites. The difference did not reach statistical significance. Upon stimulation, $\alpha-\mathrm{H} 3 \mathrm{~K} 27 \mathrm{me} 3$ signals were slightly diminished at the IL12B TSS (Figure 4F). This might reflect a decreased interaction between the TSS and the downstream putative silencer element at $+20,000 \mathrm{bp}$, where robust enrichment of $\mathrm{H} 3 \mathrm{~K} 27 \mathrm{me} 3$ was detected (Figure 4G). In summary, it seems plausible that the region IV downstream site and the TSS/ $-1,200$ bp regions are in spatial proximity to each other, and $\mathrm{H} 3 \mathrm{~K} 27 \mathrm{me} 3$ at the TSS might possibly be elevated after exposure to ascites. However, we cannot rule out that H3K27me3-dependent mechanisms are dispensable for suppression of $I L 12 B$ due to the observation that enrichments by $\alpha-\mathrm{H} 3 \mathrm{~K} 27 \mathrm{me} 3$ at the TSS was only mildly elevated in MDMs exposed to ascites relative to the control population. The identified putative regulatory elements at the $I L 12 B$ and CXCL10 loci are candidate regions for assessing binding of transcription factors that regulate $I L 12 B$ expression.

\subsection{Inducible REL and p65 Binding to Chromatin Is Not Impaired in MDMs Exposed to Ascites}

REL and p65 binding was measured (Figures 5A-C) in the same samples as in Figures 4E-G (identical data for IgG samples are shown). An enrichment with antibodies against each transcription factor was induced in the control population after stimulation at $I L 12 B-1,200$ bp (Figure 5A). Surprisingly, in the short-term ascites exposed population, REL and p65 were recruited to a similar extent at the $-1,200 \mathrm{bp}$ site of $I L 12 B$, while long-term exposure to ascites led to reduced but measurable recruitment. No recruitment was observed at the TSS and at $+20,000 \mathrm{bp}$ (Figures 5B,C). This indicates that chromatin binding of the $\mathrm{NF \kappa B}$ transcriptional activators is functional in the presence of ascites despite their strongly impaired translocation (Figure 3). Notably, at the CXCL10 locus, where REL and p65 recruitment was detected at both the TSS (Figure 5D) and an upstream element (Figure 5E), recruitment was enhanced by short-term ascites exposure but was unchanged after long-term exposure. These data show that, unexpectedly, nuclear REL and p65 levels are not measurably limiting for their binding to chromatin in MDMs exposed to ascites.

\subsection{The Role of REL in the Induction of IL12B Expression}

In order to clarify whether RNAi-mediated depletion of REL and p65 affects expression of IL12B, we employed knockdown approaches using siRNA in MDMs differentiated in normal medium. Functionality of the siRNA oligonucleotides was validated on protein level (Figure S8 in Supplementary Material). However, we were unable to achieve high knockdown efficiencies in primary macrophages, resulting in apparent protein levels of $60 \%$ relative to the control population or less. Knockdown of REL resulted in slightly diminished induction of IL-12p40 (Figure S7 in Supplementary Material). In a murine Rel knockout model, induction was largely dependent on Rel (42). However, the effects we observed after RNA interference are mild, which we attribute to incomplete depletion of the target proteins and to the effect that transfection with control siRNA also led to a strong reduction of p40 expression in most experiments. We could not find an efficient transfection reagent that did not elicit this effect, even in the absence of siRNA oligonucleotides (data not shown). In one of the MDM populations analyzed, IL12B expression was affected neither by REL knockdown nor by p65 knockdown (blue dots, Figure S7 in Supplementary Material) despite a measurable reduction of their protein levels, and in another sample, induction was increased after knockdown of p65 (black dots, Figure S7 in Supplementary Material). This prompted us to use a different experimental system in which Rel is genetically deleted. When we stimulated murine Rel knockout bone marrow-derived MDMs (BMDMs) with LPS and IFN $\gamma$, induction of Il12b (Figure 6A) as well as p40 (Figure 6B) was reduced to about $20 \%$ of the levels generated by wild-type cells, as it was similarly shown by others previously. These data suggest that REL is important but not necessary for IL12B induction. Indeed, it was observed previously by others that Rel-deficient murine antigen-presenting cells can produce IL-12p40 to a highly varying extent depending on the tissue they were isolated from Ref. (64). The function of REL is conceivably supplemented by and partially redundant with that of p65, as it was noted before $(42,65)$. The mild effects we observed after siRNA-mediated partial depletion are in line with the notion that levels of REL and p65 are not limiting for target gene induction in MDMs.

\subsection{IL-10 Is Not Sufficient to Suppress I/12b Expression}

The inability of MDMs to synthesize IL-12p40 after exposure to ascites could be explained by the negative effect of IL-10 on the translocation of REL and p65 (45-47) as well as its indirect actions via the induction of STAT3 target genes (23, 24, 66-68). However, treatment of MDMs (differentiated in normal medium) 
with recombinant IL-10 from human cells in parallel with LPS and IFN $\gamma$ did not recapitulate full suppression of IL-12p40 production (Figure 7B) and was unable to shut off $I L 12 B$ transcription
(Figure 7A) in cells from all but one donor. Importantly, the effects of partial suppression were highly significant on both mRNA (Figure 7A) and protein levels (Figure 7B), indicating

A

IL12B -1,200 bp (region III)

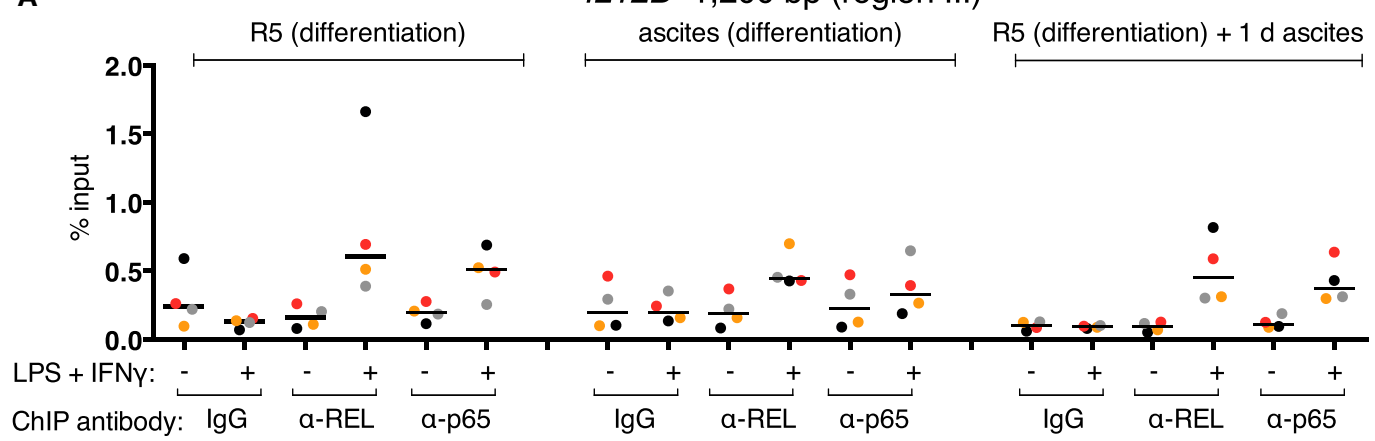

B

\section{IL 12B TSS}

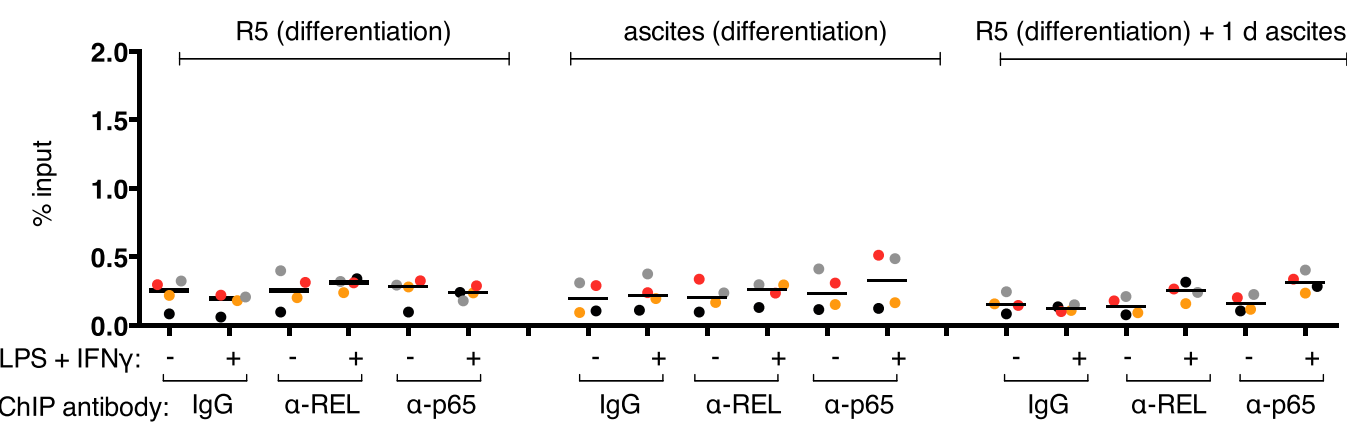

C

IL12B +20,000 bp (region IV)

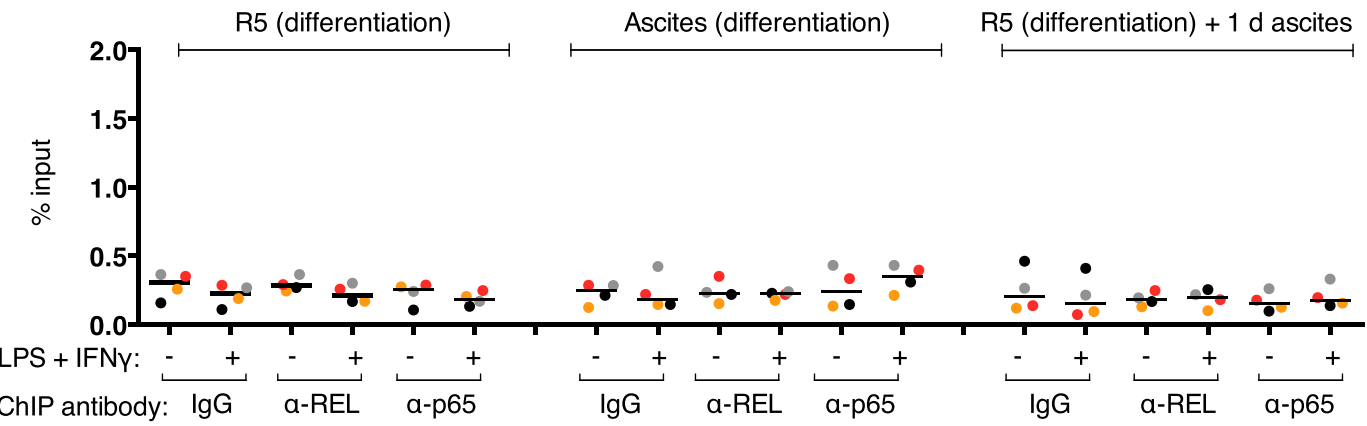

D

CXCL $10-5,000$ bp

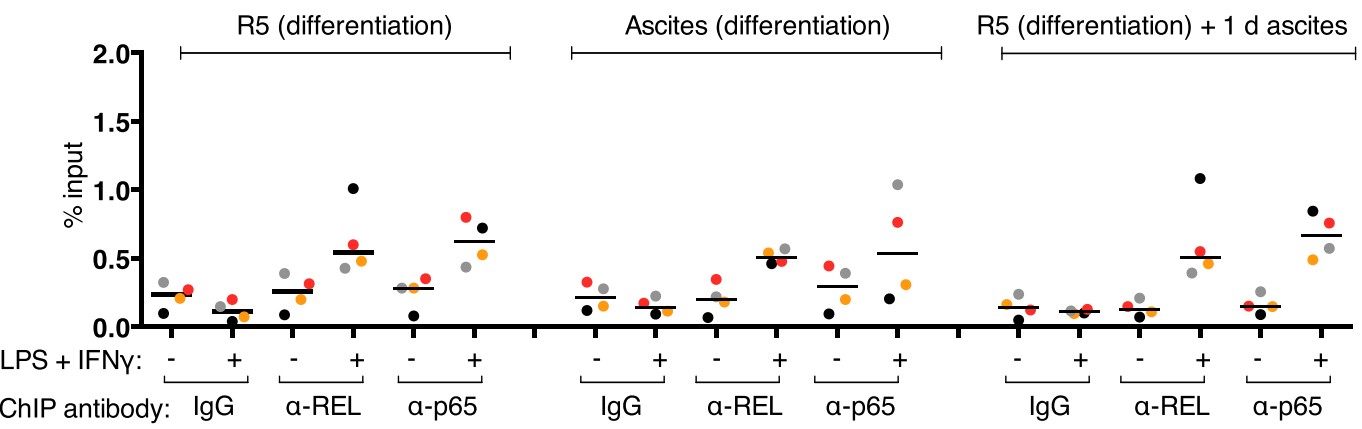

FIGURE 5 | Continued 
E CXCL10 TSS

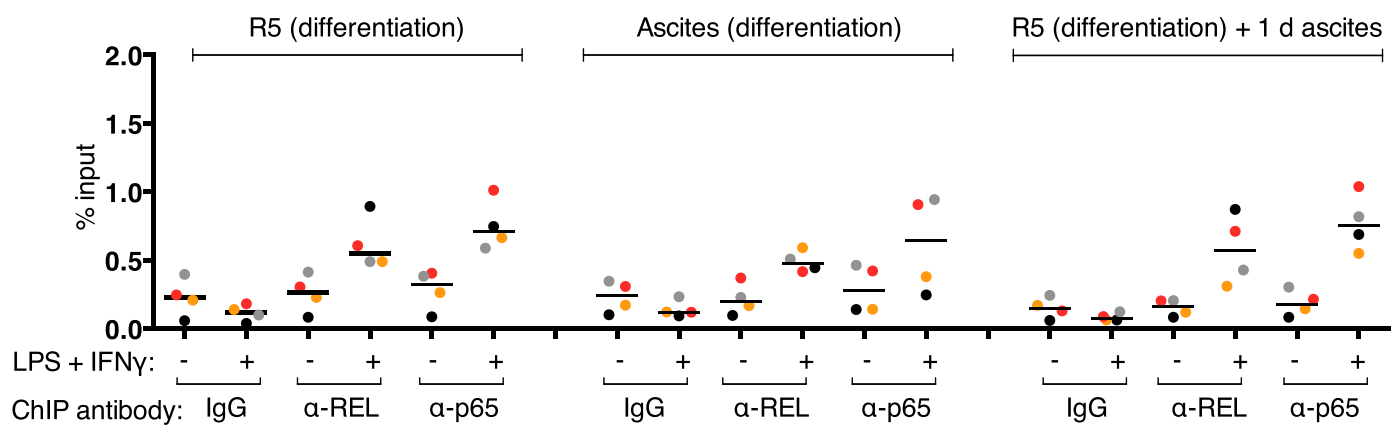

FIGURE 5 | Inducible REL and p65 binding at the IL12B and CXCL10 loci in MDMs. (A-E) ChIP-qPCR analyses of REL and p65 binding in chromatin preparations from MDMs differentiated in normal medium, in ascites, or in normal medium followed by ascites for 1 day were conducted. Cells were stimulated with or without LPS and IFN $\gamma(+)$ or their respective solvents (-) $2.5 \mathrm{~h}$ prior to harvesting. Genomic regions were amplified by specific primers are indicated. The same samples were used as in Figure $4(N=4)$. Each dot denotes a biological replicate; for each replicate, MDMs from six donors were pooled after harvesting of the cells. Median values are indicated by horizontal bars. Colors encode ascites samples from individual patients, and colors are consistent between panels within this figure, Figure 4 and Figure S9 in Supplementary Material.

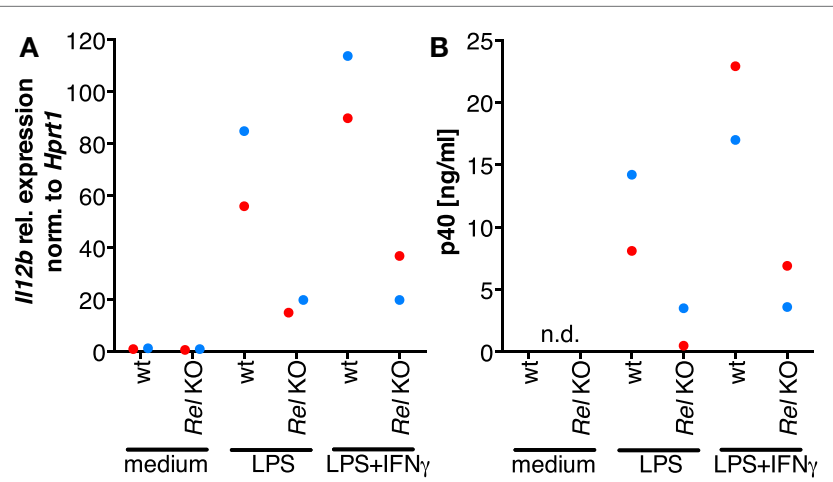

FIGURE 6 | The role of Rel in the induction of $/ / 12 b$ expression. Murine Rel knockout BMDMs or wild-type BMDMs ( $N=2$ each) were differentiated in vitro and stimulated with LPS or LPS and IFN $\gamma$ for $24 \mathrm{~h}$. Cells were used for RT-qPCR analysis of $/ 112 b$ (A), and II-12p40 was measured in supernatants by ELISA (B); n.d., not detected.

that the recombinant IL-10 was functional. MDMs from some individual donors were affected more than others, demonstrating that the amplitude of suppression is donor-dependent to a large extent. We conclude that other ascites-borne factors are necessary for full suppression of $I L 12 B$.

\section{DISCUSSION}

Our data suggest that (1) suppression of IL12B transcription by ovarian carcinoma ascites acts immediately and on differentiated macrophages, and it is reversible upon ascites withdrawal; (2) although ascites impinges on REL and p65 nuclear translocation, binding of these factors to chromatin is not diminished; and (3) IL-10 can only partially recapitulate suppression. This implicates that soluble factors in ascites may act combinatorially to achieve rapid, gene-specific suppression of $I L 12 B$ transcription.

\subsection{Reversibility and Immediacy of Ascites-Mediated IL12B Suppression}

Immediately after ascites exposure, $I L 12 B$ transcription was largely abrogated in MDMs simultaneously stimulated with LPS and IFN $\gamma$ (Figure 2A). IL12B mRNA inducibility was partially restored in cells from all donors after ascites withdrawal. These data indicate that, while suppression is rapid, its effects are not permanent in cells exposed to ascites for $24 \mathrm{~h}$. Additional posttranscriptional mechanisms negatively affecting p40 synthesis are likely (Figure 2B). Importantly, some MDM cultures retain the ability to secrete measurable amounts of $\mathrm{p} 40$ in the presence of ascites, and this apparently depends on both the donor and the ascites used. Ascites withdrawal further attenuates suppression (Figures 2A-D).

In line with reversibility of $I L 12 B$ suppression, the transcript was detected in most ex vivo TAM samples in the absence of ascites (Figure 1A). IL12B expression reached levels similar to those in TAMs cultivated in ascites in the presence of IFN $\gamma$. Furthermore, exposure to ascites does not immediately induce macrophage M2 marker expression in MDMs (Figure S3 in Supplementary Material), an observation which is compatible with a model of transient suppression by ascites.

As mentioned before, our data indicate that biological variation is high among both donor cells and ascites samples, which agrees with our finding that, on transcriptome level, TAMs from OC patients can be clustered into two groups which differ in the expression levels of interferon-responsive genes. Higher expression of these genes is positively correlated with patient survival (7). From a therapeutical perspective, it might be beneficial to determine genetic predispositions and environmental factors involved in ascites-mediated suppression of $I L 12 B$.

\subsection{Restrainment of NFKB by Ascites Is Not Complete}

Ascites-exposed MDMs show strongly reduced REL and p65 nuclear translocation (Figure 3), yet chromatin binding of 

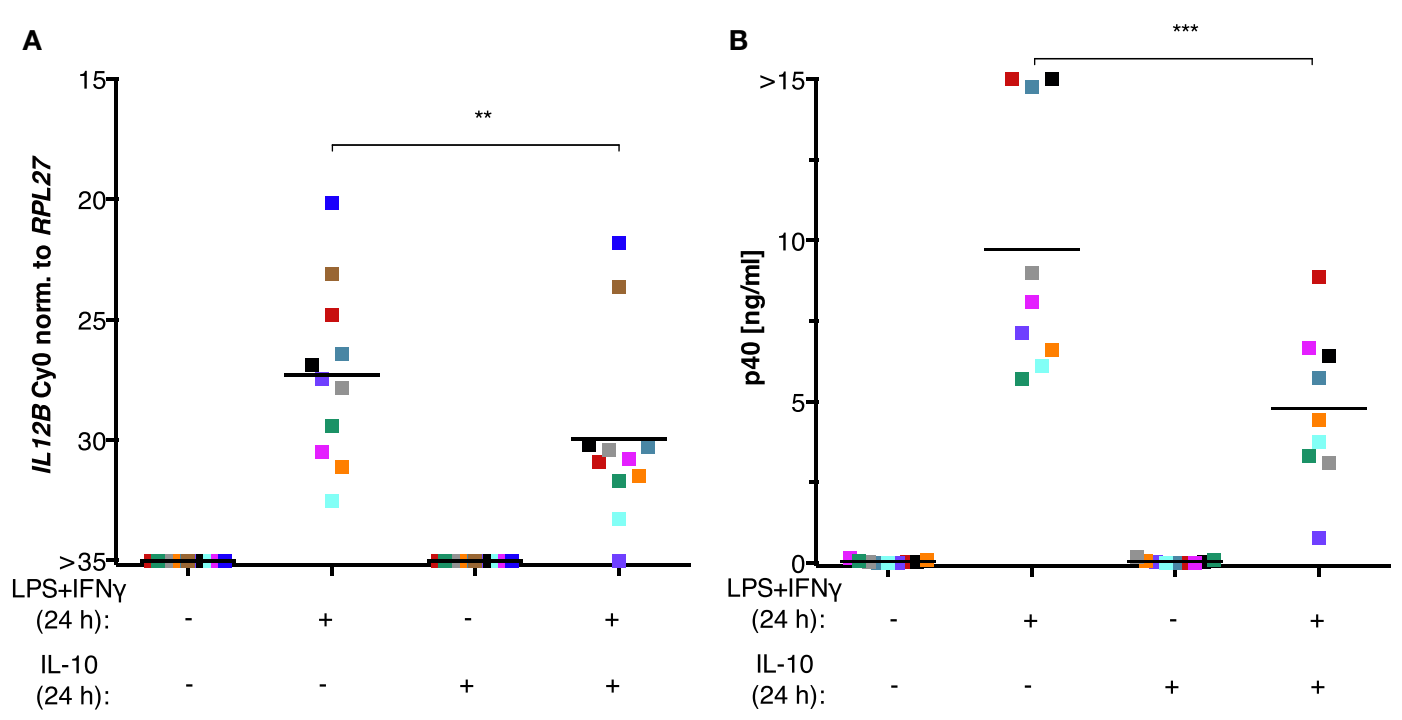

FIGURE 7 | IL-10 is not sufficient to suppress IL12B induction in MDMs. MDMs were differentiated in R5 medium for 6-7 days and stimulated with LPS and IFN $\gamma$, with recombinant IL-10 from human cells, or both for $24 \mathrm{~h}$. (A) IL $12 B$ expression was measured by RT-qPCR ( $N=11)$. (B) IL-12p40 in culture supernatants ( $N=9)$ was measured by ELISA. Each square denotes a biological replicate (individual healthy donor). Colors encode individual healthy donors, and colors are consistent between panels within this figure. Median values are indicated by horizontal bars. Statistical significances were calculated with paired $t$-tests. ${ }^{\star \star} P<0.01$; ${ }^{\star \star \star} P<0.001$.

these factors is functional (Figure 5). Additionally, after shortterm ascites exposure, REL and p65 translocation was not fully impaired in most samples (Figures 3B,D). In conclusion, nuclear REL and p65 levels after stimulus-dependent translocation are sufficient for saturation of their binding sites at the $I L 12 B$ and CXCL10 loci regardless of ascites-mediated reduction of nuclear translocation. Consistent with this, siRNA-mediated partial depletion of REL or p65 did not significantly influence IL12B induction (Figure 6A). However, it cannot be excluded that reduced binding of REL proteins to sites with lower affinity than at the $I L 12 B$ and $C X C L 10$ loci has an indirect impact on $I L 12 B$ transcription, e.g., by affecting the expression of a genespecific coactivator.

Interestingly, IL-10 was reported to selectively inhibit expression of a subset of LPS-inducible genes in murine macrophages in the absence of endogenous IL-10, and $I 112 b$ was among the most strongly downregulated transcripts (69). Gene-specific mechanisms offer a conclusive explanation why other NFkB targets such as CXCL10 are not suppressed by ascites (Figure S6 in Supplementary Material). Unaltered occupancy of REL and p65 at both the $I L 12 B$ and CXCL10 loci in the presence of ascites (Figure 5) strongly suggests that these gene-specific mechanisms do not affect NFKB chromatin binding. Induction of CXCL10 expression depends on IFN $\gamma$, its receptor and STAT1 $(70,71)$, and also on NFKB (72). These factors are likewise required for $I L 12 B$ expression. Induction of $I L 12 B$ by LPS and IFN $\gamma$ is not functional in MDMs exposed to ascites (Figure 2), while induction of CXCL10 is (Figure S6 in Supplementary Material). CXCL10 is expressed in ex vivo TAMs, while $I L 12 B$ is not (7). The two genes are presumably not regulated by differential NFKB and STAT1 recruitment to chromatin in the presence of ascites. Gene-specific posttranslational modifications of transcription factors, availability of coactivators, regulation of DNA looping involving the region IV putative silencer element, or a repressor which does not affect the marks we used to characterize the chromatin state of IL12B may be involved in suppression.

\subsection{The Role of IL-10 in IL12B Suppression}

A plethora of studies ascribe a major role to IL-10 in the maintenance of homeostasis, including the prevention of IL-12 production via multiple mechanisms $(23,24,39,43,45-47,67$, $68,73)$. Our observation that IL-10 does not fully recapitulate ascites-mediated suppression of $I L 12 B$ in vitro in cultures from most donors suggests that additional suppressive factors might be lacking. In addition to IL-10, prostaglandin E2 (73), phosphatidylserine $(74)$, adenosine $(75,76)$, lysophosphatidic acid (33), polyunsaturated fatty acids (77), and $\alpha$-fetoprotein $(78,79)$ were described to downregulate IL-12 production. Potential targets of soluble factors could be upstream signaling components, transcription factors, or cofactors.

In murine alveolar macrophages, the E3 ubiquitin ligase Trim29 was found to negatively regulate the host response after bacterial infection. Trim29, which is exclusively expressed in alveolar macrophages, can induce degradation of the Ikk regulatory subunit (80). This highlights a paradigm for tissue-specific regulation of the pro-inflammatory response. Analogously, the expression of a regulator impinging on IKK function could conceivably be modulated by soluble factors from ascites. A corepressor which suppresses Il12b expression in murine macrophages is Smrt (81). The study elucidates that the closely related proteins Ncor and Smrt can differentially repress sets of target genes. Ill2b was repressed by Smrt exclusively, and Smrt could be displaced from 
the locus by IFN $\gamma$ treatment. It is conceivable that ascites-borne soluble factors stablize SMRT or another gene-specific corepressor at the IL12B locus, since the expression of CXCL10 is not suppressed by ascites. Transcription factors described to repress IL12B expression such as Nfil3 in mice (24) or c-MAF (82) might contribute to the establishment of a locus-specific repressive complex.

Intriguingly, the levels of IL-10 as well as those of arachidonic acid in ascites are negatively correlated with patient survival according to our previous studies, and IL-10 and arachidonic acid levels are synergistically correlated with poor prognosis (21). This suggests that IL-10 alone is not sufficient to exert its full pro-tumorigenic effect in vivo. We postulate that other factors are required in addition to IL-10 for the suppression of a pro-inflammatory, antitumorigenic macrophage phenotype that includes expression of IL-12p40. Imbalances of this postulated interplay which are integrated into deregulated $I L 12 B$ expression might be involved not only in tumorigenesis but could also contribute to diseases with an autoimmune component $(26,27)$. Future studies will investigate putative combinatorial suppressive mechanisms using a panel of purified factors.

\section{DATA AVAILABILITY}

The ChIP-seq datasets generated for this study can be found in the ArrayExpress repository at https://www.ebi.ac.uk/arrayexpress/ experiments/E-MTAB-6297.

\section{ETHICS STATEMENT}

All patient samples were obtained in accordance with the recommendations of the Ethics Commission of the Department of Medicine, Philipps University of Marburg with written informed consent from all subjects. All subjects gave written informed consent in accordance with the Declaration of Helsinki. The protocol

\section{REFERENCES}

1. Bingle L, Brown NJ, Lewis CE. The role of tumour-associated macrophages in tumour progression: implications for new anticancer therapies. J Pathol (2002) 196(3):254-65. doi:10.1002/path.1027

2. Condeelis J, Pollard JW. Macrophages: obligate partners for tumor cell migration, invasion, and metastasis. Cell (2006) 124:263-6. doi:10.1016/j.cell. 2006.01.007

3. Santin AD, Bellone S, Ravaggi A, Roman J, Smith CV, Pecorelli S, et al. Increased levels of interleukin-10 and transforming growth factor- $\beta$ in the plasma and ascitic fluid of patients with advanced ovarian cancer. BJOG (2001) 108:804-8. doi:10.1111/j.1471-0528.2001.00206.x

4. Worzfeld T, Pogge von Strandmann E, Huber M, Adhikary T, Wagner U, Reinartz S, et al. The unique molecular and cellular microenvironment of ovarian cancer. Front Oncol (2017) 7:24. doi:10.3389/fonc.2017.00024

5. Reinartz S, Schumann T, Finkernagel F, Wortmann A, Jansen JM, Meissner W, et al. Mixed-polarization phenotype of ascites-associated macrophages in human ovarian carcinoma: correlation of CD163 expression, cytokine levels and early relapse. Int J Cancer (2013) 134(1):32-42. doi:10.1002/ijc.28335

6. Finkernagel F, Reinartz S, Lieber S, Adhikary T, Wortmann A, Hoffmann N, et al. The transcriptional signature of human ovarian carcinoma macrophages is associated with extracellular matrix reorganization. Oncotarget (2016) 7(46):75339-52. doi:10.18632/oncotarget.12180 was approved by the Ethics Commission of the Department of Medicine, Philipps University of Marburg.

\section{AUTHOR CONTRIBUTIONS}

AU performed most experiments. FF applied all bioinformatical methods. TA performed TAM ChIPs, MIRA assays, and ChIPseq library preparation. NH performed IL12B induction kinetics analysis. BJ and FN performed ELISA experiments. AN performed high-throughput sequencing. UW contributed clinical samples. SR prepared TAM samples. AV performed all experiments with murine cells. AU, FF, AV, SR, SM-B, RM, and TA conceived and designed the experiments. All authors analyzed data. RM and TA supervised the study. TA wrote the manuscript. AU, SM-B, and RM made major contributions to the writing of the manuscript.

\section{ACKNOWLEDGMENTS}

We thank Bernhard Wilke, Klaus Weber, Alexandra Schneider, Angelika Filmer, Margitta Alt, Achim Allmeroth, and Traute Plaum for expert technical support. This work was published as part of the doctoral thesis work of Annika Unger (née Wortmann) (83).

\section{FUNDING}

The study was supported by grants from the Universiätsklinikum Marburg und Giessen (01/2015 to TA and UW), from the DFG (RE 1590/1-1 to SR and MU 601/17-1 to RM), and from the Wilhelm-Sander-Stiftung (2016.123.1 to SM-B).

\section{SUPPLEMENTARY MATERIAL}

The Supplementary Material for this article can be found online at https://www.frontiersin.org/articles/10.3389/fimmu.2018.01425/ full\#supplementary-material.

7. Adhikary T, Wortmann A, Finkernagel F, Lieber S, Nist A, Stiewe T, et al. Interferon signaling in ascites-associated macrophages is linked to a favorable clinical outcome in a subgroup of ovarian carcinoma patients. BMC Genomics (2017) 18(1):243. doi:10.1186/s12864-017-3630-9

8. Yuan X, Zhang J, Li D, Mao Y, Mo F, Du W, et al. Prognostic significance of tumor-associated macrophages in ovarian cancer: a meta-analysis. Gynecol Oncol (2017) 147(1):181-7. doi:10.1016/j.ygyno.2017.07.007

9. Schneemann M, Schoedon G, Hofer S, Blau N, Guerrero L, Schaffner A. Nitric oxide synthase is not a constituent of the antimicrobial armature of human mononuclear phagocytes. J Infect Dis (1993) 167(6):1358-63. doi:10.1093/ infdis/167.6.1358

10. Konur A, Krause SW, Rehli M, Kreutz M, Andreesen R. Human monocytes induce a carcinoma cell line to secrete high amounts of nitric oxide. J Immunol (1996) 157(5):2109-15.

11. Fang FC. Antimicrobial reactive oxygen and nitrogen species: concepts and controversies. Nat Rev Microbiol (2004) 2:820-32. doi:10.1038/nrmicro1004

12. Schneemann M, Schoeden G. Macrophage biology and immunology: man is not a mouse. J Leukoc Biol (2007) 81:579; discussion 580. doi:10.1189/jlb. 1106702

13. Gross TJ, Kremens K, Powers LS, Brink B, Knutson T, Domann FE, et al. Epigenetic silencing of the human NOS2 gene: rethinking the role of nitric oxide in human macrophage inflammatory responses. J Immunol (2014) 192(5):2326-38. doi:10.4049/jimmunol.1301758 
14. Balakrishnan A, Schnare M, Chakravortty D. Of men not mice: bactericidal/ permeability-increasing protein expressed in human macrophages acts as a phagocytic receptor and modulates entry and replication of Gram-negative bacteria. Front Immunol (2016) 7:455. doi:10.3389/fimmu.2016.00455

15. Anzai R, Seki S, Ogasawara K, Hashimoto W, Sugiura K, Satoh M, et al. Interleukin-12 induces cytotoxic NK1 Ag+ $\alpha \beta \mathrm{T}$ cells in the lungs of euthymic and athymic mice. Immunology (1996) 88:82-9. doi:10.1046/j.1365-2567.1996. d01-638.x

16. Takeda K, Seki S, Ogasawara A, Anzai R, Hashimoto W, Sugiura K, et al. Liver NK1.1+CD4+ T cells activated by IL-12 as a major effector in inhibition of experimental tumor metastasis. J Immunol (1996) 156:3366-73.

17. Cui J, Shin T, Kawano T, Sato H, Kondo E, Toura I, et al. Requirement for V $\alpha 14$ NKT cells in IL-12-mediated rejection of tumors. Science (1997) 278:1623-6. doi:10.1126/science.278.5343.1623

18. Kawamura T, Takeda K, Mendiratta SK, Kawamura H, Kaer LV, Yagita H, et al. Critical role of NK1+ T cells in IL-12-induced immune responses in vivo. J Immunol (1998) 160:16-9.

19. Kodama T, Takeda K, Shimozato O, Hayakawa Y, Atsuta M, Kobayashi K, et al. Perforin-dependent NK cell cytotoxicity is sufficient for anti-metastatic effect of IL-12. Eur J Immunol (1999) 29:1390-6. doi:10.1002/(SICI)15214141(199904)29:04<1390::AID-IMMU1390>3.0.CO;2-C

20. Gotlieb WH, Abrams JS, Watson JM, Velu TJ, Berek JS, Martinez-Maza O. Presence of interleukin 10 (IL-10) in the ascites of patients with ovarian and other intra-abdominal cancers. Cytokine (1992) 4(5):385-90. doi:10.1016/ 1043-4666(92)90082-3

21. Reinartz S, Finkernagel F, Adhikary T, Rohnalter V, Schumann T, Schober Y, et al. A transcriptome-based global map of signaling pathways in the ovarian cancer microenvironment associated with clinical outcome. Genome Biol (2016) 17:108. doi:10.1186/s13059-016-0956-6

22. Sica A, Saccani A, Bottazzi B, Polentarutti N, Vecchi A, van Damme J, et al. Autocrine production of IL-10 mediates defective IL-12 production and NF-kappa B activation in tumor-associated macrophages. J Immunol (2000) 164(2):762-7. doi:10.4049/jimmunol.164.2.762

23. Aste-Amezaga M, Ma X, Sartori A, Trinchieri G. Molecular mechanisms of the induction of IL-12 and its inhibition by IL-10. J Immunol (1998) 160:5936-44.

24. Smith A, Qualls J, O'Brien K, Balouzian L, Johnson P, Schultz-Cherry S, et al. A distal enhancer in Il12b is the target of transcriptional repression by the STAT3 pathway and requires the basic leucine zipper (B-ZIP) protein NFIL3. J Biol Chem (2011) 286(26):23582-90. doi:10.1074/jbc.M111.249235

25. D’Andrea A, Rengaraju M, Valiante NM, Chehimi J, Kubin M, Aste M, et al. Production of natural killer cell stimulatory factor (NKSF/IL-12) by peripheral blood mononuclear cells. J Exp Med (1992) 176(5):1387-98. doi:10.1084/ jem.176.5.1387

26. Segal BM, Dwyer BK, Shevach EM. An interleukin (IL)-10/IL-12 immunoregulatory circuit controls susceptibility to autoimmune disease. J Exp Med (1998) 187(4):537-46. doi:10.1084/jem.187.4.537

27. Ma X, Yan W, Zheng H, Du Q, Zhang L, Ban Y, et al. Regulation of IL-10 and IL-12 production and function in macrophages and dendritic cells. F1000Res (2015) 4:1465. doi:10.12688/f1000research.7010.1

28. Buechler C, Ritter M, Orsó E, Langmann T, Klucken J, Schmitz G. Regulation of scavenger receptor $\mathrm{CD} 163$ expression in human monocytes and macrophages by pro- and antiinflammatory stimuli. J Leukoc Biol (2000) 67(1):97-103. doi:10.1002/jlb.67.1.97

29. Sulahian TH, Högger P, Wahner AE, Wardwell K, Goulding NJ, Sorg C, et al. Human monocytes express CD163, which is upregulated by IL-10 and identical to p155. Cytokine (2000) 12(9):1312-21. doi:10.1006/ cyto. 2000.0720

30. Philippidis P, Mason JC, Evans BJ, Nadra I, Taylor KM, Haskard DO, et al. Hemoglobin scavenger receptor CD163 mediates interleukin-10 release and heme oxygenase-1 synthesis. Circ Res (2004) 94:119-26. doi:10.1161/01.RES. 0000109414.78907.F9

31. Hsieh CS, Macatonia SE, Tripp CS, Wolf S, O'Garra A, Murphy KM. Development of CD4+ T cells through IL-12 production by Listeria-induced macrophages. Science (1993) 260:547-9. doi:10.1126/science.8097338

32. Handel-Fernandez ME, Cheng X, Herbert LM, Lopez DM. Down-regulation of IL-12, not a shift from a Thelper-1 to a T helper-2 phenotype, is responsible for impaired IFN-gamma production in mammary tumor-bearing mice. J Immunol (1997) 158(1):280-6.
33. Michel T, Hentges F, Zimmer J. Consequences of the crosstalk between monocytes/macrophages and natural killer cells. Front Immunol (2013) 3: 403. doi:10.3389/fimmu.2012.00403

34. Duluc D, Corvaisier M, Blanchard S, Catala L, Descamps P, Gamelin E, et al. Interferon-gamma reverses the immunosuppressive and protumoral properties and prevents the generation of human tumor-associated macrophages. Int J Cancer (2009) 125(2):367-73. doi:10.1002/ijc.24401

35. Beatty G, Chiorean E, Fishman M, Saboury B, Teitelbaum U, Sun W, et al. CD40 agonists alter tumor stroma and show efficacy against pancreatic carcinoma in mice and humans. Science (2011) 331:1612-6. doi:10.1126/science.1198443

36. Rosenzweig $S$, Holland $S$. Defects in the interferon- $\gamma$ and interleukin-12 pathways. Immunol Rev (2005) 203:38-47. doi:10.1111/j.0105-2896.2005. 00227.x

37. Bustamante J, Picard C, Boisson-Dupuis S, Abel L, Casanova J-L. Genetic lessons learned from X-linked Mendelian susceptibility to mycobacterial diseases. Ann N Y Acad Sci (2011) 1246:92-101. doi:10.1111/j.1749-6632.2011.06273.x

38. Bustamante J, Boisson-Dupuis S, Abel L, Casanova J-L. Mendelian susceptibility to mycobacterial disease: genetic, immunological, and clinical features of inborn errors of IFN- $\gamma$ immunity. Semin Immunol (2014) 26:454-70. doi:10.1016/j.smim.2014.09.008

39. Ma X, Chow J, Gri G, Carra G, Gerosa G, Wolf S, et al. The interleukin 12 p40 gene promoter is primed by interferon $\gamma$ in monocytic cells. J Exp Med (1996) 183:147-57. doi:10.1084/jem.183.1.147

40. Mason N, Aliberti J, Caamano JC, Liou H-C, Hunter CA. Identification of $c$-Rel-dependent and -independent pathways of IL-12 production during infectious and inflammatory stimuli. J Immunol (2002) 168:2590-4. doi:10.4049/ jimmunol.168.6.2590

41. Visekruna A, Volkov A, Steinhoff U. A key role for NF-kB transcription factor $c$-Rel in T-lymphocyte-differentiation and effector functions. Clin Dev Immunol (2012) 2012:239368. doi:10.1155/2012/239368

42. Sanjabi S, Williams K, Saccani S, Zhou L, Hoffmann A, Ghosh G, et al. A c-Rel subdomain responsible for enhanced DNA-binding affinity and selective gene activation. Genes Dev (2005) 19:2138-51. doi:10.1101/gad.1329805

43. Rahim SS, Khan N, Boddupalli CS, Hasnain SE, Mukhopadhyay S. Interleukin-10 (IL-10) mediated suppression of IL-12 production in RAW 264.7 cells also involves c-rel transcription factor. Immunology (2005) 114(3):313-21. doi:10.1111/j.1365-2567.2005.02107.x

44. Saccani A, Schioppa T, Porta C, Biswas SK, Nebuloni M, Vago L, et al. p50 nuclear factor-kappaB overexpression in tumor-associated macrophages inhibits M1 inflammatory responses and antitumor resistance. Cancer Res (2006) 66: 11432-40. doi:10.1158/0008-5472.CAN-06-1867

45. Wang P, Wu P, Siegel MI, Egan RW, Billah MM. IL-10 inhibits transcription of cytokine genes in human peripheral blood mononuclear cells. J Immunol (1994) 153(2):811-6.

46. Schottelius AJG, Mayo MW, Balfour Sartor R, Baldwin ASJ. Interleukin-10 signaling blocks inhibitor of $\mathrm{kB}$ kinase activity and nuclear factor $\mathrm{kB}$ DNA binding. J Biol Chem (1999) 274:31868-74. doi:10.1074/jbc.274.45.31868

47. Lentsch AB, Shanley TP, Sarma V, Ward PA. In vivo suppression of NF-kappa $\mathrm{B}$ and preservation of I kappa B alpha by interleukin-10 and interleukin-13. J Clin Invest (1997) 100(10):2443-8. doi:10.1172/JCI119786

48. Guescini M, Sisti D, Rocchi M, Stocchi L, Stocchi V. A new real-time PCR method to overcome significant quantitative inaccuracy due to slight amplification inhibition. BMC Bioinformatics (2008) 9:326. doi:10.1186/1471-2105-9-326

49. Adhikary T, Wortmann A, Schumann T, Finkernagel F, Lieber S, Roth K, et al. The transcriptional PPAR $\beta / \delta$ network in human macrophages defines a unique agonist-induced activation state. Nucleic Acids Res (2015) 43(10):5033-51. doi:10.1093/nar/gkv331

50. Adhikary T, Müller R. In vivo studies of PPAR-chromatin interactions: chromatin immunoprecipitation for single-locus and genomewide analyses. Methods Mol Biol (2013) 952:175-85. doi:10.1007/978-1-62703-155-4_12

51. Saccani S, Pantano S, Natoli G. Two waves of nuclear factor $\mathrm{kb}$ recruitment to target promoters.J ExpMed (2001) 193(12):1351. doi:10.1084/jem.193.12.1351

52. Worzfeld T, Finkernagel F, Reinartz S, Konzer A, Adhikary T, Nist A, et al. Proteotranscriptomics reveal signaling networks in the ovarian cancer microenvironment. Mol Cell Proteomics (2018) 17(2):270-89. doi:10.1074/ mcp.RA117.000400

53. Brown K, Park S, Kanno T, Franzoso G, Siebenlist U. Mutual regulation of the transcriptional activator NF-kappa B and its inhibitor, I kappa B-alpha. Proc Natl Acad Sci U S A (1993) 90(6):2532-6. doi:10.1073/pnas.90.6.2532 
54. Bunting K, Rao S, Hardy K, Woltring D, Denyer GS, Wang J, et al. Genomewide analysis of gene expression in T cells to identify targets of the NF-kB transcription factor c-Rel. J Immunol (2007) 178:7097-109. doi:10.4049/ jimmunol.178.11.7097

55. Chin M, Herscovitch M, Zhang N, Waxman DJ, Gilmore TD. Overexpression of an activated REL mutant enhances the transformed state of the human B-lymphoma BJAB cell line and alters its gene expression profile. Oncogene (2009) 28:2100-11. doi:10.1038/onc.2009.74

56. van Essen D, Zhu Y, Saccani S. A feed-forward circuit controlling inducible NF- $\mathrm{B}$ target gene activation by promoter histone demethylation. Mol Cell (2010) 39(5):750-60. doi:10.1016/j.molcel.2010.08.010

57. Clarke DL, Clifford RL, Jindarat S, Proud D, Pang L, Belvisi M, et al. TNF $\alpha$ and IFN- $\gamma$ synergistically enhance transcriptional activation of CXCL10 in human airway smooth muscle cells via STAT-1, NF- $\kappa B$, and the transcriptional coactivator CREB-binding protein. J Biol Chem (2020) 285:29101-10. doi:10.1074/jbc.M109.099952

58. Piunti A, Shilatifard A. Epigenetic balance of gene expression by polycomb and COMPASS families. Science (2016) 352(6290):aad9780. doi:10.1126/ science.aad 9780

59. Kim T-Y, Shiekhattar R. Diverse regulatory interactions of long noncoding RNAs. Curr Opin Genet Dev (2016) 36:73-82. doi:10.1016/j.gde.2016.03.014

60. Huang D-B, Chen Y-Q, Ruetsche M, Phelps CB, Ghosh G. X-ray crystal structure of proto-oncogene product c-Rel bound to the CD28 response element of IL-2. Structure (2001) 9(8):669-78. doi:10.1016/S0969-2126(01)00635-9

61. Barski A, Cuddapah S, Cui K, Roh T-Y, Schones DE, Wang Z, et al. Highresolution profiling of histone methylations in the human genome. Cell (2007) 129:823-37. doi:10.1016/j.cell.2007.05.009

62. Jung M, Kadam S, Xiong W, Rauch TA, Jin S-G, Pfeifer GP. MIRA-seq for DNA methylation analysis of CpG islands. Epigenomics (2015) 7(5):695-706. doi:10.2217/epi.15.33

63. Kuo H-C, Lin P-Y, Chung T-C, Chao C-M, Lai L-C, Tsai M-H, et al. DBCAT: database of $\mathrm{CpG}$ islands and analytical tools for identifying comprehensive methylation profiles in cancer cells. J Comput Biol (2011) 18(8):1013-7. doi:10.1089/cmb.2010.0038

64. Hilliard BA, Mason N, Xu L, Sun J, Lamhamedi-Cherradi S-E, Liou H-C, et al. Critical roles of c-Rel in autoimmune inflammation and helper $\mathrm{T}$ cell differentiation. J Clin Invest (2002) 110(6):843-50. doi:10.1172/JCI0215254

65. Sanjabi S, Hoffmann A, Liou H-C, Baltimore D, Smale ST. Selective requirement for c-Rel during IL-12 P40 gene induction in macrophages. Proc Natl Acad Sci U S A (2000) 97(23):12705-10. doi:10.1073/pnas.230436397

66. Stearns M, Kim G, Garcia F, Wang M. Interleukin-10 induced activating transcription factor 3 transcriptional suppression of matrix metalloproteinase-2 gene expression in human prostate CPTX-1532 cells. Mol Cancer Res (2004) 2(7):403-16

67. Gilchrist M, Thorsson V, Li B, Rust A, Korb M, Roach J, et al. Systems biology approaches identify ATF3 as a negative regulator of toll-like receptor 4. Nature (2006) 441:173-8. doi:10.1038/nature04768

68. Whitmore M, Iparraguirre A, Kubelka L, Weninger W, Hai T, Williams B. Negative regulation of TLR-signaling pathways by activating transcription factor-3. J Immunol (2007) 179:3622-30. doi:10.4049/jimmunol.179.6.3622

69. Lang R, Patel D, Morris JJ, Rutschman RL, Murray P. Shaping gene expression in activated and resting primary macrophages by IL-10. J Immunol (2002) 169:3353-63. doi:10.4049/jimmunol.169.5.2253

70. Ramana CV, Gil MP, Han Y, Ransohoff R, Schreiber RD, Stark GR. Stat1independent regulation of gene expression in response to IFN- $\gamma$. Proc Natl Acad Sci U S A (2001) 98(12):6674-9. doi:10.1073/pnas.111164198
71. de Paus RA, Kilic SS, van Dissel JT, van de Vosse E. Effect of amino acid substitutions in the human IFN- $\gamma \mathrm{R} 2$ on IFN- $\gamma$ responsiveness. Genes Immun (2011) 12:136-44. doi:10.1038/gene.2010.74

72. Tamassia N, Calzetti F, Ear T, Cloutier A, Gasperini S, Bazzoni F, et al. Molecular mechanisms underlying the synergistic induction of CXCL10 by LPS and IFN- $\gamma$ in human neutrophils. Eur J Immunol (2007) 37:2627-34. doi:10.1002/eji.200737340

73. van der Pouw Kraan TC, Boeije LC, Smeenk RJ, Wijdenes J, Aarden LA. Prostaglandin-E2 is a potent inhibitor of human interleukin 12 production. J Exp Med (1995) 181:775-9. doi:10.1084/jem.181.2.775

74. Link A, Kino T, Worth JA, McGuire JL, Crane ML, Chrousos GP, et al. Ligandactivation of the adenosine A2a receptors inhibits IL-12 production by human monocytes. J Immunol (2000) 164(1):436-42. doi:10.4049/jimmunol.164.1.436

75. Haskó G, Kuhel DG, Chen JF, Schwarzschild MA, Deitch EA, Mabley JG, et al. Adenosine inhibits IL-12 and TNF- $\alpha$ production via adenosine A2a receptordependent and independent mechanisms. FASEB J (2000) 14(13):2065-74. doi:10.1096/fj.99-0508com

76. Panther E, Idzko M, Corinti S, Ferrari D, Herouy Y, Mockenhaupt M, et al. The influence of lysophosphatidic acid on the functions of human dendritic cells. J Immunol (2002) 169(8):4129-35. doi:10.4049/jimmunol.169.8.4129

77. Zhang M, Fritsche KL. Fatty acid-mediated inhibition of IL-12 production by murine macrophages is independent of PPARgamma. Br J Nutr (2004) 91(5):733-9. doi:10.1079/BJN20041096

78. Um SH, Mulhall C, Alisa A, Ives AR, Karani J, Williams R, et al. $\alpha$-Fetoprotein impairs APC function and induces their apoptosis. J Immunol (2004) 173(3):1772-8. doi:10.4049/jimmunol.173.3.1772

79. Yamamoto M, Tatsumi T, Miyagi T, Tsunematsu H, Aketa H, Hosui A, et al. $\alpha$-Fetoprotein impairs activation of natural killer cells by inhibiting the function of dendritic cells. Clin Exp Immunol (2011) 165(2):211-9. doi:10.1111/j.1365-2249.2011.04421.x

80. Xing J, Weng L, Yuan B, Wang Z, Jia L, Jin R, et al. Identification of a role for TRIM29 in the control of innate immunity in the respiratory tract. Nat Immunol (2016) 17(12):1373-80. doi:10.1038/ni.3580

81. Ghisletti S, Huang W, Jepsen K, Benner C, Hardiman G, Rosenfeld MG, et al. Cooperative NCoR/SMRT interactions establish a corepressor-based strategy for integration of inflammatory and anti-inflammatory signaling pathways. Genes Dev (2009) 23(6):681-93. doi:10.1101/gad.1773109

82. Cao S, Liu J, Chesi M, Bergsagel PL, Ho I-C, Donnelly RP, et al. Differential regulation of IL-12 and IL-10 gene expression in macrophages by the basic leucine zipper transcription factor c-Maf fibrosarcoma. J Immunol (2002) 169(19):5715-25. doi:10.4049/jimmunol.169.10.5715

83. Unger A. Suppression proinflammatorischer Gene in Makrophagen durch Aszites des Ovarialkarzinoms [PhD thesis]. Marburg: Philipps University of Marburg (2017).

Conflict of Interest Statement: The authors declare that the research was conducted in the absence of any commercial or financial relationships that could be construed as a potential conflict of interest.

Copyright (c) 2018 Unger, Finkernagel, Hoffmann, Neuhaus, Joos, Nist, Stiewe, Visekruna, Wagner, Reinartz, Müller-Brüsselbach, Müller and Adhikary. This is an open-access article distributed under the terms of the Creative Commons Attribution License (CC BY). The use, distribution or reproduction in other forums is permitted, provided the original author(s) and the copyright owner are credited and that the original publication in this journal is cited, in accordance with accepted academic practice. No use, distribution or reproduction is permitted which does not comply with these terms. 